\title{
A Generalisation of AGM Contraction and Revision to Fragments of First-Order Logic
}

\author{
Zhiqiang Zhuang \\ College of Intelligence and Computing \\ Tianjin University, China
}

Zhe Wang

ZHE.WANG@GRIFFITH.EDU.AU

School of Information Communication Technology

Griffith University, Australia

Kewen Wang

School of Information Communication Technology

Griffith University, Australia

ZHUANG@TJU.EDU.CN

James Delgrande

JIM@CS.SFU.CA

School of Computing Science

Simon Fraser University, Canada

\begin{abstract}
AGM contraction and revision assume an underlying logic that contains propositional logic. Consequently, this assumption excludes many useful logics such as the Horn fragment of propositional logic and most description logics. Our goal in this paper is to generalise AGM contraction and revision to (near-)arbitrary fragments of classical first-order logic. To this end, we first define a very general logic that captures these fragments. In so doing, we make the modest assumptions that a logic contains conjunction and that information is expressed by closed formulas or sentences. The resulting logic is called first-order conjunctive logic or FC logic for short. We then take as the point of departure the AGM approach of constructing contraction functions through epistemic entrenchment, that is the entrenchment-based contraction. We redefine entrenchment-based contraction in ways that apply to any FC logic, which we call FC contraction. We prove a representation theorem showing its compliance with all the AGM contraction postulates except for the controversial recovery postulate. We also give methods for constructing revision functions through epistemic entrenchment which we call FC revision; which also apply to any FC logic. We show that if the underlying FC logic contains tautologies then FC revision complies with all the AGM revision postulates. Finally, in the context of FC logic, we provide three methods for generating revision functions via a variant of the Levi Identity, which we call contraction, withdrawal and cut generated revision, and explore the notion of revision equivalence. We show that withdrawal and cut generated revision coincide with FC revision and so does contraction generated revision under a finiteness condition.
\end{abstract}

\section{Introduction}

The area of belief change studies how an intelligent agent may change its beliefs rationally in the face of new information. The dominant theory in belief change is the AGM framework (Alchourrón, Gärdenfors, \& Makinson, 1985; Gärdenfors, 1988) which focuses on two kinds of change, contraction, in which an agent retracts some beliefs, and revision, in which an agent consistently incorporates some information into its set of beliefs. In the AGM 
framework belief change is expressed at the knowledge level; in particular, an agent's beliefs are modelled by a logically closed set of formulas called a belief set.

The AGM framework has a minimal requirement on the underlying logic, that it is at least as expressive as classical propositional logic. Hence the underlying logic must fully support all the logical connectives in propositional logic such as negation and disjunction. This requirement limits the applicability of the framework, since many artificial intelligence applications are based on logical languages that lack certain logical connectives of propositional logic. Generally, such logics intends to trade off expressivity in favour of better computational behaviour, and consequently they are often better suited for practical applications. To remedy this limitation, significant effort has been made on constructing AGM-style contraction and revision functions for individual logics lack certain logical connectives of propositional logic.

To date, the focus of such research has been on fragments of propositional logic and description logics (e.g., Booth, Meyer, Varzinczak, \& Wassermann, 2011; Delgrande \& Peppas, 2015; Delgrande \& Wassermann, 2013; Creignou, Papini, Pichler, \& Woltran, 2014; Wang, Wang, \& Topor, 2015a; Zhuang, Wang, Wang, \& Qi, 2014, 2016). The guiding principle of these works is that the proposed contraction or revision functions maintain, as well as possible, the AGM approach. Various techniques are developed to address the inexpressiveness of the logic fragment, while attempting to adhere to the AGM approach. What is common about these works is that they focus on a particular logic and develop techniques specifically for that logic. However, given the vast number of inapplicable logics, it is not practical to deal with all of them individually. In this paper, we develop techniques that work for (near-)arbitrary fragments of first-order logic.

Our strategy is to define a logic, called first-order conjunctive logic or FC logic for short, which is general enough to subsume many of the inapplicable ones, and construct contraction and revision functions for this general logic. Then these belief change functions will automatically apply to any of the subsumed logics. Concretely, a logic is an FC logic if it is a fragment of first-order logic (a first-order fragment for short) that supports conjunction. Furthermore, we assume that an agent's beliefs and formulas for belief change are expressed via closed formulas only. ${ }^{1}$ Arguably, FC logic subsumes all first-order fragments that are of practical importance including, but not limited to, all fragments of propositional logic (propositional fragments for short) and most description logics (Baader, Calvanese, McGuinness, Nardi, \& Patel-Schneider, 2003).

Of the various (equivalent) construction methods for belief contraction, we focus on entrenchment-based contraction (Gärdenfors \& Makinson, 1988). Intuitively, an agent's beliefs vary in their epistemic importance, and a rational agent will give up a less important belief over a more important one in a contraction. Thus all formulas are ranked by their epistemic importance, where higher-ranked formulas are deemed more important. The outcome of a contraction is then determined by using this ranking on formulas. In particular, the agent believes $\psi$ after the contraction by $\phi$ if the agent originally believes $\psi$ and the disjunction $\phi \vee \psi$ is higher in the ranking than $\phi$.

We start by redefining entrenchment-based contraction for FC logic, which we call $F C$ contraction. To get a grasp of the problem at hand, note that entrenchment-based contrac-

1. A first-order logic formula is closed if all variables in the formula are quantified. 
tion refers to disjunctions of formulas, but FC logic in general does not support disjunction. The key in our approach is to avoid a reliance on any logical connective within a logic fragment. We provide two techniques for such avoidance which relies on the new notion of FC approximations and the existing notion of cut (Williams, 1994) respectively, and which turn out to have the same effect. We provide a representation theorem for FC contraction which shows that it possesses all properties of AGM contraction except for the controversial recovery postulate.

Moving on to revision, again we give a construction method that applies to $\mathrm{FC}$ logic in general, which we call $F C$ revision. It is well-known that, as for contraction functions, revision functions can also be constructed through a ranking over all formulas (Peppas, 2008). In particular, an agent believes $\psi$ after the revision by $\phi$ if the disjunction $\neg \phi \vee \psi$ is higher in the ranking than $\neg \phi$. FC revision is obtained by redefining this construction method for revision functions. The problem then is the inability to represent the disjunction $\neg \phi \vee \psi$ and the negation $\neg \phi$ by all FC logics. The same techniques as for FC contraction are used for avoiding the reliance on such disjunction and negation. FC revision is well-behaved, as we can show it possesses all properties of AGM revision, assuming that the underlying FC logic supports tautologies.

Moreover, for revision, we provide three methods for constructing revision functions via a variant of the Levi identity (Levi, 1991), namely contraction generated revision, withdrawal generated revision, and cut generated revision, which apply to FC logic in general. In the AGM framework, revision functions can be constructed through contraction functions via the Levi identity. The construction consists of two steps, a contraction step which removes the negation of the revising formula from the belief set then an expansion step which adds the revising formula to the belief set that resulted from the contraction step. The catch here is that FC logic, in general, does not support negation, thus we have to redefine the contraction step. Again this is achieved with the notion of FC approximations and that of cut. We can show that withdrawal and cut generated revision coincide with FC revision, and contraction generated revision coincide with FC revision under a finiteness condition.

The rest of this paper is organised as follows. We first present FC logic in the next section, this is followed by an overview of the AGM framework, emphasising entrenchmentbased contraction. Then the details of FC contraction, FC revision, and contraction, withdrawals and cut generated revision are given in Section 4, 5 and 6 respectively. Finally, we discuss the related work and conclude the paper. Proofs of results are given in the appendices.

\section{First-Order Conjunctive Logic}

We adopt the Tarskian definition of logics, under which a logic is a pair $\langle\mathcal{L}, C n\rangle$ where $\mathcal{L}$ is the underlying language and $C n: 2^{\mathcal{L}} \rightarrow 2^{\mathcal{L}}$ is a function that takes each subset of $\mathcal{L}$ to another. The intention is that $\operatorname{Cn}(X)$ consists of all logical consequences of $X$. Under this setting, propositional and classical first-order logic are denoted $\left\langle\mathcal{L}_{\mathrm{P}}, C n_{\mathrm{P}}\right\rangle$ and $\left\langle\mathcal{L}_{\mathrm{F}}, C n_{\mathrm{F}}\right\rangle$ respectively. Throughout this paper, propositional atoms are written as $p, q, \ldots$, formulas as $\phi, \psi, \ldots$, and sets of formulas as $S, X, \ldots$ The letter $K$ is reserved to represent a belief set (i.e., a set $X$ such that $X=C n(X))$ in some understood logic. 
A logic $\left\langle\mathcal{L}^{\prime}, C n^{\prime}\right\rangle$ is a fragment of a logic $\langle\mathcal{L}, C n\rangle$ if $\mathcal{L}^{\prime} \subseteq \mathcal{L}$ and $C n^{\prime}(X)=C n(X) \cap$ $\mathcal{L}^{\prime}$ for all $X \subseteq \mathcal{L}^{\prime}$. As a simple example, by considering propositional atoms as nullary predicates, propositional logic can be seen as a fragment of first-order logic. Major fragments of propositional logic include the (propositional) Horn and Krom fragments. The Horn fragment, denoted $\left\langle\mathcal{L}_{\mathrm{H}}, C n_{\mathrm{H}}\right\rangle$, allows only clauses with at most one positive literal, and conjunctions of these clauses. The Krom fragment, denoted $\left\langle\mathcal{L}_{\mathrm{K}}, C n_{\mathrm{K}}\right\rangle$, allows only clauses with at most two literals and conjunctions of these clauses. Although some description logics have features that are not covered in first-order logic, most description logics are fragments of first-order logic. For instance, DL-Lite ${ }_{\text {core }}$ (Calvanese, De Giacomo, Lembo, Lenzerini, \& Rosati, 2007) is a first-order fragment that allows only formulas of the form

$$
A(a), \quad R(a, b), \quad \forall x[C(x) \rightarrow D(x)], \quad \text { and } \quad \forall x[C(x) \rightarrow \neg D(x)]
$$

where $C(x)$ and $D(x)$ are of the form

$$
A(x), \quad \exists y R(x, y) \quad \text { or } \quad \exists y R(y, x)
$$

for $A$ a unary predicate; $R$ a binary predicate; $a, b$ constants; and $x, y$ variables.

The following defines those fragments of first-order logic that we deal with.

Definition 1. A logic $\langle\mathcal{L}, C n\rangle$ is a first-order conjunctive logic (FC logic) if

1. $\mathcal{L} \subseteq \mathcal{L}_{\mathrm{F}}$

2. $C n(X)=C n_{\mathrm{F}}(X) \cap \mathcal{L}$ for all $X \subseteq \mathcal{L}$; and

3. $\mathcal{L}$ is closed under conjunction, that is, $\phi, \psi \in \mathcal{L}$ implies $(\phi \wedge \psi) \in \mathcal{L}$.

For generality, the class of $\mathrm{FC}$ logics is intentionally defined to be vague regarding the supported logical connectives other than conjunction. That is, an FC logic may or may not support connectives like disjunction, negation or implication, but it must support conjunction. Conjunction is required in an FC logic so that we can describe crucial properties of contraction and revision involving the conjunction of formulas. ${ }^{2}$ We emphasise that supporting conjunction is hardly an expressiveness requirement. Since the most natural interpretation regarding $\left\{\phi_{1}, \ldots, \phi_{n}\right\}$ is as having the same truth value as $\phi_{1} \wedge \cdots \wedge \phi_{n}$, if a first-order fragment were to not explicitly support conjunction, we could still represent the conjunction $\phi_{1} \wedge \cdots \wedge \phi_{n}$ in the fragment by the set $\left\{\phi_{1}, \ldots, \phi_{n}\right\}$. So, arguably, any first-order fragment supports conjunction, at least implicitly.

In fact we can discard conjunction completely by working with multiple contractions and revisions (Fuhrmann \& Hansson, 1994; Peppas, 2008). Multiple contractions can be interpreted as aiming to remove some or all formulas in a set; thus contraction by $\phi \wedge \psi$ is equivalent to contraction by $\{\phi, \psi\}$. Multiple revisions can be interpreted as aiming to incorporate all formulas in a set; thus revision by $\phi \wedge \psi$ is equivalent to revision by $\{\phi, \psi\} .^{3}$ The downside of working with multiple contractions and revisions is that we have to change

2. These are the supplementary postulates for contraction and revision in the AGM framework.

3. Note that under the other interpretations of multiple contractions and revisions such as those in (Delgrande \& Jin, 2012), contraction and revision by a set of formulas cannot be reduced to contraction and revision by a conjunction of formulas. 
most of the AGM notations that have become the standard. For the sake of readability, we decided to go with conjunction.

We add a further requirement, which we henceforth assume, that the formulas in an agent's belief set and the formulas involved in belief change are closed, which means they have no unquantified variables. This is a minimal restriction, given that it is a standard assumption in logical approaches in Artificial Intelligence. ${ }^{4}$ By the definition of FC logic and the above consideration, all propositional fragments and all description logics that are first-order fragments are FC logics.

For any FC logic $\left\langle\mathcal{L}_{\mathrm{FC}}, C n_{\mathrm{FC}}\right\rangle$ we write $X \vdash_{\mathrm{FC}} \phi$ to denote $\phi \in C n_{\mathrm{FC}}(X)$ and $\vdash_{\mathrm{FC}} \phi$ to denote $\phi \in C n_{\mathrm{FC}}(\emptyset)$, and we abbreviate $C n_{\mathrm{FC}}(\{\phi\})$ as $C n_{\mathrm{FC}}(\phi)$. We may omit the subscript for $\vdash$ if it is clear from the context. A formula or set of formulas under $\left\langle\mathcal{L}_{\mathrm{FC}}, C n_{\mathrm{FC}}\right\rangle$ is consistent if it is consistent under the standard definition of consistency for first-order logic. We denote the (unique) inconsistent belief set, which consists of $\mathcal{L}_{\mathrm{FC}}$, as $K_{\perp}$.

To some extent, all FC logics, except for first-order logic, are limited in expressivity such that there are certain first-order formulas they cannot represent. For instance, description logics do not allow disjunctions of axioms. It is then useful to have the following notion of FC approximations which captures the approximations of the inexpressible first-order formulas as formulas in the underlying FC logic.

Definition 2. Let $\left\langle\mathcal{L}_{\mathrm{FC}}, C n_{\mathrm{FC}}\right\rangle$ be an $F C$ logic and $\phi$ a first-order formula. Then the set of $\mathrm{FC}$ approximations of $\phi$ with respect to $\left\langle\mathcal{L}_{\mathrm{FC}}, C n_{\mathrm{FC}}\right\rangle$, denoted $\mathcal{F C}\left(\phi, \mathcal{L}_{\mathrm{FC}}\right)$, is such that

$$
\mathcal{F C}\left(\phi, \mathcal{L}_{\mathrm{FC}}\right)=\left\{\psi \in \mathcal{L}_{\mathrm{FC}} \mid \psi \vdash_{\mathrm{F}} \phi\right\} .
$$

An FC approximation of a first-order formula $\phi$ with respect to an FC logic $\left\langle\mathcal{L}_{\mathrm{FC}}, C n_{\mathrm{FC}}\right\rangle$ is a formula in $\mathcal{L}_{\mathrm{FC}}$ that entails $\phi$ under first-order logic. Note that we abbreviate $\mathcal{F C}\left(\phi, \mathcal{L}_{\mathrm{FC}}\right)$ as $\mathcal{F C}(\phi)$ whenever the underlying FC logic is clear from the context. Consider the clause $\neg p \vee q \vee r$. This clause is not part of the Horn fragment as it has more than one positive literal. It is also not part of the Krom fragment as it has more than two literals. If the underlying logic is the Horn fragment of propositional logic, then

$$
\mathcal{F C}\left(\neg p \vee q \vee r, \mathcal{L}_{\mathrm{H}}\right)=\{\perp, \neg p, q, r, \neg p \vee q, \neg p \vee r\} .
$$

If instead, the underlying logic is the Krom fragment; then, since disjunctions with two positive literals are allowed, $q \vee r$ is also an FC approximation, that is

$$
\mathcal{F C}\left(\neg p \vee q \vee r, \mathcal{L}_{\mathrm{K}}\right)=\{\perp, \neg p, q, r, \neg p \vee q, \neg p \vee r, q \vee r\} .
$$

Note that, the set of FC approximation can be empty. For instance, let the FC logic $\left\langle\mathcal{L}_{\mathrm{FC}}, C n_{\mathrm{FC}}\right\rangle$ be a propositional fragment such that $\mathcal{L}_{\mathrm{FC}}=\{a, b, a \wedge b, \top\}$. Since $\left\langle\mathcal{L}_{\mathrm{FC}}, C n_{\mathrm{FC}}\right\rangle$ does not support negation nor $\perp$, none of the formulas in $\mathcal{L}_{\mathrm{FC}}$ entails the negation of any formula in $\mathcal{L}_{\mathrm{FC}}$, that is $\mathcal{F C}\left(\neg a, \mathcal{L}_{\mathrm{FC}}\right)=\emptyset, \mathcal{F C}\left(\neg b, \mathcal{L}_{\mathrm{FC}}\right)=\emptyset$, and so on. To another extreme, the set of FC approximation can be infinite. ${ }^{5}$

In many situations, it is useful to work with the logically weakest FC approximations, which we call the closest FC approximations.

4. we note that approaches that appear to employ free variables most often take such variables to be implicitly universally quantified. Hence the clause $\operatorname{Mother}(x, y) \wedge \operatorname{Parent}(y, z) \rightarrow \operatorname{Grandmother}(x, z)$ would in fact stand for $\forall x, y, z[\operatorname{Mother}(x, y) \wedge \operatorname{Parent}(y, z) \rightarrow \operatorname{Grandmother}(x, z)]$.

5. We give one such example in Appendix A. 
Definition 3. Let $\left\langle\mathcal{L}_{\mathrm{FC}}, C n_{\mathrm{FC}}\right\rangle$ be an $F C$ logic and $\phi$ a first-order formula. Then the set of Closest FC approximations of $\phi$ with respect to $\left\langle\mathcal{L}_{\mathrm{FC}}, C n_{\mathrm{FC}}\right\rangle$, denoted $\mathcal{C} \mathcal{F C}\left(\phi, \mathcal{L}_{\mathrm{FC}}\right)$, is such that

$$
\mathcal{C F C}\left(\phi, \mathcal{L}_{\mathrm{FC}}\right)=\left\{\psi \in \mathcal{F C}\left(\phi, \mathcal{L}_{\mathrm{FC}}\right) \mid \psi^{\prime} \in \mathcal{F C}\left(\phi, \mathcal{L}_{\mathrm{FC}}\right) \text { and } \psi^{\prime} \vdash_{\mathrm{FC}} \psi \text { imply } \psi \vdash_{\mathrm{FC}} \psi^{\prime}\right\}
$$

For the above examples, we have

$$
\mathcal{C F C}\left(\neg p \vee q \vee r, \mathcal{L}_{\mathrm{H}}\right)=\{\neg p \vee q, \neg p \vee r\}
$$

and

$$
\mathcal{C F C}\left(\neg p \vee q \vee r, \mathcal{L}_{\mathrm{K}}\right)=\{\neg p \vee q, \neg p \vee r, q \vee r\} .
$$

Given an FC logic $\left\langle\mathcal{L}_{\mathrm{FC}}, C n_{\mathrm{FC}}\right\rangle$ and a first-order formula $\phi$, if $\mathcal{F C}\left(\phi, \mathcal{L}_{\mathrm{FC}}\right)$ is empty, then $\mathcal{C F C}\left(\phi, \mathcal{L}_{\mathrm{FC}}\right)$ is obviously so, however $\mathcal{C} \mathcal{F C}\left(\phi, \mathcal{L}_{\mathrm{FC}}\right)$ could be empty, even though $\mathcal{F C}\left(\phi, \mathcal{L}_{\mathrm{FC}}\right)$ is not. ${ }^{6}$

The notion of FC approximations is not completely new. A similar concept is given for the Horn fragment which is called Horn strengthening (Kautz \& Selman, 1996). For any formula $\phi$ in $\mathcal{L}_{\mathrm{P}}$, its set of Horn strengthenings consists of logically the weakest formulas in $\mathcal{L}_{\mathrm{H}}$ that entail $\phi$. Hence for any such $\phi$, its set of Horn strengthenings is exactly the set of closest FC approximations of $\phi$ with respect to $\left\langle\mathcal{L}_{\mathrm{H}}, C n_{\mathrm{H}}\right\rangle$.

\section{The AGM Framework for Belief Change}

The area of belief change (Gärdenfors, 1988; Peppas, 2008) studies how an agent may change its belief state given new information. The best-known, and indeed central, approach to belief change is the AGM framework (Alchourrón et al., 1985; Gärdenfors, 1988) of Alchourron, Gärdenfors, and Makinson. In the AGM framework, the goal is to describe belief change at the knowledge level, that is on an abstract level, independent of how beliefs are represented and manipulated. The two main belief change functions considered are belief contraction, in which an agent ceases to believe a formula $\phi$, and belief revision, in which an agent adopts a new belief given by a formula $\phi$.

In this framework, belief states are modelled by sets of sentences, called belief sets, closed under the logical consequence operator of a compact logic that includes classical propositional logic and satisfies the deduction theorem. Throughout this section let the logic be $\langle\mathcal{L}, C n\rangle$. Thus a belief set $K$ satisfies the constraint:

If $K$ logically entails $\phi$ then $\phi \in K$.

Consequently, if $K$ is a belief set then $K=\operatorname{Cn}(K)$, where $\operatorname{Cn}(K)$ is the deductive closure of $K$. Contraction and revision are modelled as functions from belief sets and formulas to belief sets. In addition, the operation $K+\phi$ is the expansion of $K$ by $\phi$, and is defined as $C n(K \cup\{\phi\})$. Expansion captures the simplest form of belief change; it can be reasonably applied when the new information is consistent with the current set of beliefs.

In belief change, a change operator is typically addressed by two different means. First, a set of rationality postulates that characterise a belief change function can be defined.

6. We give one such example in Appendix A. 
Second, a formal construction can be defined to specify a class of change functions. Ideally, the set of rationality postulates is then shown to exactly capture the same class of functions as those determined by the given construction.

Consider first belief contraction. Contraction addresses the situation in which an agent loses information. Informally, the contraction of a belief set by a formula is another belief set in which that formula is not believed. Formally, a contraction function $\dot{-}$ is a function from $2^{\mathcal{L}} \times \mathcal{L}$ to $2^{\mathcal{L}}$ satisfying the following postulates.

$(K \dot{-} 1) K \dot{-} \phi$ is a belief set.

$(K \dot{-} 2) \quad K \dot{-} \phi \subseteq K$.

$(K \dot{-} 3)$ If $\phi \notin K$ then $K \dot{-} \phi=K$.

$(K \dot{-} 4)$ If $\forall \phi$ then $\phi \notin K \dot{-} \phi$.

$(K \dot{-} 5)$ If $\phi \in K$ then $K \subseteq(K \dot{-} \phi)+\phi$.

$(K \dot{-} 6)$ If $\operatorname{Cn}(\phi)=C n(\psi)$ then $K \dot{-} \phi=K \dot{-} \psi$.

$(K \dot{-} 7) K \dot{-} \phi \cap K \dot{-} \psi \subseteq K \dot{-}(\phi \wedge \psi)$.

$(K \dot{-} 8)$ If $\psi \notin K \dot{-}(\psi \wedge \phi)$ then $K \dot{-}(\phi \wedge \psi) \subseteq K \dot{-} \psi$.

Thus, contraction is meaningful only for believed sentences $(K \dot{-} 3)$ and yields a belief set $(K \dot{-})$ in which the sentence for contraction $\phi$ is not believed (unless $\phi$ is a tautology) $(K \dot{-} 4)$ and no new sentences are believed $(K \dot{-} 2)$. The fifth postulate, the so-called recovery postulate, states that nothing is lost if one contracts and expands by the same sentence. This postulate is controversial; see Hansson's work (1999) for a discussion. The sixth postulate asserts that contraction is independent of how a sentence is expressed. The last two postulates express relations between contracting by a conjunction and the constituent conjuncts. Hence $(K \dot{-} 7)$ says that if a formula is in the result of contracting by each of two formulas then it is in the result of contracting by their conjunction. $(K \dot{-} 8)$ says that if a conjunct is not in the result of contracting by a conjunction, then contracting by that conjunct is (using $(K \dot{-} 7))$ the same as contracting by the conjunction.

Note that, in the presence of the other postulates, $(K \dot{-} 7)$ is equivalent to the postulate of conjunctive trisection (Rott, 1992; Hansson, 1993).

$$
(K \dot{-} c t) \text { If } \phi \in K \dot{-}(\phi \wedge \psi) \text {, then } \phi \in K \dot{-}(\phi \wedge \psi \wedge \delta) \text {. }
$$

$(K \dot{-} c t)$ is well motivated. Informally speaking, it says that if $\phi$ is the more preferred of a pair of formulas $\{\phi, \psi\}$, it is not the least preferred in the increased set of three formulas $\{\phi, \psi, \delta\}$. In a contraction, it is rational to discard the less important formulas whenever possible, thus $\phi$ being retained in contracting by $\phi \wedge \psi$ means $\phi$ is more important than $\psi$. Since $\phi$ is more important than $\psi$, it is not the least important formula among $\phi, \psi$, and $\delta$. Thus, $\phi$ should be retained in contracting by $\phi \wedge \psi \wedge \delta$.

Several (equivalent) construction methods have been proposed for contraction. Here we review the classic method called entrenchment-based contraction. As noted, the beliefs held by an agent are not equal in terms of their epistemic importance. According to 
Gärdenfors and Makinson (1988), more important beliefs are said to be more entrenched, and the relative entrenchments between formulas is modelled by a relation called epistemic entrenchment. Given a belief set $K$, an epistemic entrenchment associated with $K$ is a binary relation $\leq$ over the underlying language such that $\phi \leq \psi$ means $\psi$ is at least as entrenched as $\phi$. The strict relation $\phi<\psi$ is defined as $\phi \leq \psi$ and $\psi \not \leq \phi$. Importantly, $\leq$ satisfies the following conditions:

(EE1) If $\phi \leq \psi$ and $\psi \leq \sigma$, then $\phi \leq \sigma$.

(EE2) If $\phi \vdash \psi$, then $\phi \leq \psi$.

(EE3) $\phi \leq \phi \wedge \psi$ or $\psi \leq \phi \wedge \psi$.

(EE4) If $K$ is consistent, then $\phi \notin K$ iff $\phi \leq \psi$ for all $\psi$.

(EE5) If $\phi \leq \psi$ for every $\phi$, then $\vdash \psi$.

Thus an epistemic entrenchment is a transitive relation $(E E 1)$ such that logically stronger formulas are not more entrenched than weaker ones (EE2) (and so logically equivalent formulas are equally entrenched); a conjunction is as entrenched as its least entrenched conjunct $(E E 2)-(E E 3)$; non-beliefs are least entrenched $(E E 4)$; and tautologies are most entrenched (EE5).

Entrenchment-based contraction functions are then defined via the following condition, where $\leq$ is an epistemic entrenchment associated with $K$.

$$
(C \dot{-}) \psi \in K \dot{-} \phi \text { iff } \psi \in K \text { and either } \phi<\phi \vee \psi \text { or } \vdash \phi \text {. }
$$

Thus $\psi$ is believed by the agent after the contraction by $\phi$ (i.e., $\psi \in K \dot{-} \phi$ ) if and only if it was originally believed (i.e., $\psi \in K$ ) and there is "sufficient evidence" for retaining it (i.e., $\phi<\phi \vee \psi$ ), or it is not possible to remove $\phi$ (i.e., $\vdash \phi$ ).

Given a contraction function $\dot{-}: 2^{\mathcal{L}} \times \mathcal{L} \mapsto 2^{\mathcal{L}}$, we can obtain an epistemic entrenchment $\leq$ through the following condition:

$$
(C \leq) \phi \leq \psi \text { iff } \phi \notin K \dot{-} \phi \wedge \psi \text { or } \vdash \phi \wedge \psi .
$$

It is shown that entrenchment-based contraction functions are fully characterised by $(K \dot{-} 1)-$ $(K \dot{-8})$ (Gärdenfors \& Makinson, 1988).

In contrast to belief contraction, belief revision represents the situation in which new information is to be incorporated into an agent's beliefs. Since this information may be inconsistent with the agent's beliefs, some beliefs may need to be dropped in order to obtain a consistent belief set. Formally, a revision function ${ }^{*}$ is a function from $2^{\mathcal{L}} \times \mathcal{L}$ to $2^{\mathcal{L}}$ satisfying the following postulates.

$(K * 1) K * \phi$ is a belief set.

$(K * 2) \phi \in K * \phi$.

$(K * 3) K * \phi \subseteq K+\phi$.

$(K * 4)$ If $\neg \phi \notin K$ then $K+\phi \subseteq K * \phi$. 
$(K * 5) K * \phi=K_{\perp}$ iff $\vdash \neg \phi$.

$(K * 6)$ If $\operatorname{Cn}(\phi)=\operatorname{Cn}(\psi)$ then $K * \phi=K * \psi$.

$(K * 7) K *(\phi \wedge \psi) \subseteq(K * \phi)+\psi$.

$(K * 8)$ If $\neg \psi \notin K * \phi$ then $(K * \phi)+\psi \subseteq K *(\phi \wedge \psi)$.

That is: the result of revising $K$ by $\phi$ is a belief set $(K * 1)$ in which $\phi$ is believed $(K * 2)$; whenever the result is consistent, revision consists of the expansion of $K$ by $\phi(K * 3)-$ $(K * 4)$; the only time that the inconsistent belief set, $K_{\perp}$, is obtained is when $\neg \phi$ is a tautology $(K * 5)$; and revision is independent of the syntactic form of $\phi(K * 6)$. The last two postulates deal with the relation between revising with a conjunction and expansion: when consistent, revision by a conjunction is equivalent to revision by a conjunct followed by expansion by the other conjunct.

As with contraction, constructions of revision functions have also been defined. The best known are Grove's system of spheres (Grove, 1988), which builds on Lewis' work on counterfactuals (Lewis, 1973), and the faithful rankings of Katsuno and Mendelzon (Katsuno \& Mendelzon, 1992). A revision function can also be constructed in a similar way to entrenchment-based contraction (Peppas, 2008). For convenience, we call it entrenchmentbased revision. Entrenchment-based revision functions are defined via the following condition, where $\leq$ is an epistemic entrenchment associated with $K$.

$(R *) \quad \psi \in K * \phi$ iff either $\neg \phi<\neg \phi \vee \psi$ or $\vdash \neg \phi$.

Thus $\psi$ is believed after the revision of $K$ by $\phi$ (i.e., $\psi \in K * \phi$ ) if and only if there is "sufficient evidence" (i.e., $\neg \phi<\neg \phi \vee \psi$ ) for believing it or the revision fails (i.e., $\vdash \neg \phi)$. Entrenchment-based revision functions are fully characterised by $(K * 1)-(K * 8)$.

Apart from the classical constructions, revision can be naturally defined in terms of contraction by means of the Levi Identity:

$$
K * \phi=(K \dot{-} \neg \phi)+\phi .
$$

Thus, to revise by $\phi$, make $K$ consistent with $\phi$ by contracting $K$ by $\neg \phi$, then expand the contraction outcome by $\phi$. In fact, conversely, contraction can be defined in terms of revision by means of Harper Identity (Harper, 1976):

$$
K-\phi=(K * \neg \phi) \cap K .
$$

\section{FC Contraction}

Entrenchment-based contraction is not applicable to all FC logics, as the condition $(C \dot{-})$ refers to the disjunction $\phi \vee \psi$ that is not supported by all FC logics. In this section, we provide a version of entrenchment-based contraction that is applicable to all FC logics. Unless explicitly stated, we work with an indefinite FC logic, denoted $\left\langle\mathcal{L}_{\mathrm{FC}}, C n_{\mathrm{FC}}\right\rangle$, in the remainder of the paper.

We first fix the relative entrenchments between formulas of the $\mathrm{FC}$ logic $\left\langle\mathcal{L}_{\mathrm{FC}}, C n_{\mathrm{FC}}\right\rangle$. The relative entrenchments are represented by a binary relation over $\mathcal{L}_{\mathrm{FC}}$ that satisfies the $\left\langle\mathcal{L}_{\mathrm{FC}}, C n_{\mathrm{FC}}\right\rangle$ version of conditions $(E E 1)-(E E 5)$ which can be obtained from $(E E 1)-(E E 4)$ 
by assuming $\mathcal{L}_{\mathrm{FC}}$ as the underlying language and $C n_{\mathrm{FC}}$ as the consequence operator. As an FC logic may not be able to represent tautologies, the version for (EE5) which is shown below states that the condition takes effect only when the logic can represent tautologies.

$(E E 5)$ If there is $\delta \in \mathcal{L}_{\mathrm{FC}}$ such that $\vdash_{\mathrm{FC}} \delta$, then $\phi \leq \psi$ for all $\phi$ implies $\vdash_{\mathrm{FC}} \psi$.

We call such a binary relation an $F C$ epistemic entrenchment.

Next we reformulate $(C \dot{-})$ so that it can be applied in $\left\langle\mathcal{L}_{\mathrm{FC}}, C n_{\mathrm{FC}}\right\rangle$. The obstacle here is the disjunction $\phi \vee \psi$, which is not necessarily in $\mathcal{L}_{\mathrm{FC}}$. We provide two methods to get around the difficulty, firstly by working with the FC approximations of $\phi \vee \psi$ with respect to $\left\langle\mathcal{L}_{\mathrm{FC}}, C n_{\mathrm{FC}}\right\rangle$ and secondly by utilising the notion of cut (Williams, 1994).

The first method gives rise to the following reformulated version of $(C \dot{-})$.

$\left(C_{\mathrm{FC}} \dot{\dot{-}}\right) \quad \psi \in K \dot{\dot{ }} \phi$ iff $\psi \in K$ and either there is $\delta \in \mathcal{F C}(\phi \vee \psi)$ such that $\phi<\delta$ or $\vdash_{\mathrm{F}} \phi$.

According to $\left(C_{\mathrm{FC}} \dot{-}\right)$, to decide whether $\psi$ is in $K \dot{-} \phi$, we first check if $\psi$ is in $K$ and if so we compare the relative entrenchment between $\phi$ and the FC approximations of $\phi \vee \psi$. The existence of an FC approximation being strictly more entrenched than $\phi$ is a sufficient condition for retaining $\psi$ in $K \dot{-} \phi$. Similar to $(C \dot{-})$, another sufficient condition is that $\phi$ is a tautology.

In fact, it suffices to consider only the closest $\mathrm{FC}$ approximations of $\phi \vee \psi$, whenever they exist; that is, given that $\mathcal{C F C}(\phi \vee \psi)$ is non-empty, the condition obtained by replacing $\mathcal{F C}(\phi \vee \psi)$ with $\mathcal{C F C}(\phi \vee \psi)$ in $\left(C_{\mathrm{FC}} \dot{-}\right)$ is equivalent to $\left(C_{\mathrm{FC}} \dot{-}\right)$. This is because if there is a $\delta \in \mathcal{F C}(\phi \vee \psi)$ such that $\phi<\delta$, then there must be a $\delta^{\prime} \in \mathcal{C} \mathcal{F C}(\phi \vee \psi)$ such that $\delta \vdash_{\mathrm{FC}} \delta^{\prime}$. It then follows from $(E E 2)$ that $\delta \leq \delta^{\prime}$ and from which we have by $(E E 1)$ that $\phi<\delta^{\prime}$. Conversely, if there is a $\delta^{\prime} \in \mathcal{C F C}(\phi \vee \psi)$ such that $\phi<\delta^{\prime}$, then since $\delta^{\prime} \in \mathcal{F C}(\phi \vee \psi)$, there is a $\delta \in \mathcal{F C}(\phi \vee \psi)$ such that $\phi<\delta$.

The second method is inspired by the notion of cut and its usage in defining the belief base version of entrenchment-based contraction (Williams, 1994). Although proposed in the context of propositional logic, the notion can be easily generalised to all FC logics. Following Williams (1994), the cut for a first-order formula $\phi$ with respect to a belief set $K$ and an associated FC epistemic entrenchment $\leq$ is the set $c u t_{\leq}(\phi)$ obtained as follows:

$$
\psi \in \text { cut }_{\leq}(\phi) \text { iff } \psi \in K \text { and }\{\delta \in K \mid \psi \leq \delta\} \nvdash_{\mathrm{F}} \phi .
$$

Note that a cut can be obtained for any first-order formula. It follows immediately from the definition that $\mathrm{cut}_{\leq}(\phi)$ consists of all formulas that are strictly more entrenched than $\phi$ whenever $\phi$ is in $\mathcal{L}_{\mathrm{FC}}$, and cut $_{\leq}(\phi)=K$ whenever $K \nvdash_{\mathrm{F}} \phi$. More importantly, we observe that, in the AGM setting, $\phi<\phi \vee \psi$ if and only if $c u t_{\leq}(\phi) \vdash_{\mathrm{F}} \phi \vee \psi$. This gives rise to the following reformulated version of $(C \dot{-})$.

$$
\left(C_{\mathrm{FC}}^{\text {cut }} \dot{-}\right) \psi \in K \dot{-} \phi \text { iff } \psi \in K \text { and either } c u t_{\leq}(\phi) \vdash_{\mathrm{F}} \phi \vee \psi \text { or } \vdash_{\mathrm{F}} \phi
$$

Rather than looking for an FC approximation of $\phi \vee \psi$ that is strictly more entrenched than $\phi$, with $\left(C_{\mathrm{FC}}^{c u t} \dot{-}\right)$, we check if the cut of $\phi$ entails $\phi \vee \psi$ under first-order logic. Actually, we can show that there exists one such FC approximation if and only if the entailment holds, which leads to the equivalence of $\left(C_{\mathrm{FC}} \dot{\dot{ }}\right)$ and $\left(C_{\mathrm{FC}}^{c u t} \dot{-}\right)$. 
Proposition 1. Let $\leq$ be an FC epistemic entrenchment for $K$. Then cut $_{\leq}(\phi) \vdash_{\mathrm{F}} \phi \vee \psi$ iff there is $\delta \in \mathcal{F C}(\phi \vee \psi)$ such that $\phi<\delta$.

Formally, the version of entrenchment-based contraction for $\left\langle\mathcal{L}_{\mathrm{FC}}, C n_{\mathrm{FC}}\right\rangle$, called $F C$ contraction, is defined as follows.

Definition 4. A function $\dot{-}: 2^{\mathcal{L}_{\mathrm{FC}}} \times \mathcal{L}_{\mathrm{FC}} \mapsto 2^{\mathcal{L}_{\mathrm{FC}}}$ is an $F C$ contraction function if $K \dot{-} \phi$ is determined by an $F C$ epistemic entrenchment associated with $K$ via $\left(C_{\mathrm{FC}} \dot{-}\right)$.

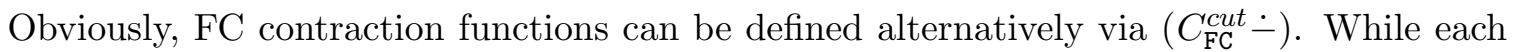
of the alternatives is particularly useful in proving certain properties of FC contraction, a significant advantage of $\left(C_{\mathrm{FC}}^{c u t}-\dot{)}\right)$ is the avoidance of computing $\mathrm{FC}$ approximations for the disjunction $\phi \vee \psi$, which can be infinitely many. Hence it provides a viable option for implementing FC contraction functions.

Following the AGM tradition, we will make explicit some properties of FC contraction by proving a representation theorem for it. The proof is more involved than that of the representation theorem for entrenchment-based contraction, since we no longer have a functionally complete set of logical connectives at our disposal. As a preparatory result, we can show that the FC epistemic entrenchment generated from an FC contraction function $\dot{-}$ via $(C \leq)$ is exactly the one that determines $\dot{-}$.

Theorem 1. If $\dot{-}$ is an $F C$ contraction function for which $K \dot{-} \phi$ is determined by the $F C$ epistemic entrenchment $\leq$ associated with $K$, then - satisfies $(C \leq)$.

To prove this theorem and the next two, note that if disjunctions of formulas are allowed, then the proof can go through as in the AGM setting. The key point is that we can indirectly refer to disjunctions via the link provided in the definition of FC approximations. That is, FC approximations of $\phi \vee \psi$ entail $\phi \vee \psi$ under first-order logic.

Next, we present the representation theorem.

Theorem 2. A function $-: 2^{\mathcal{L}_{\mathrm{FC}}} \times \mathcal{L}_{\mathrm{FC}} \mapsto 2^{\mathcal{L}_{\mathrm{FC}}}$ is an FC contraction function iff $\dot{-}$ satisfies the following postulates: ${ }^{7}$

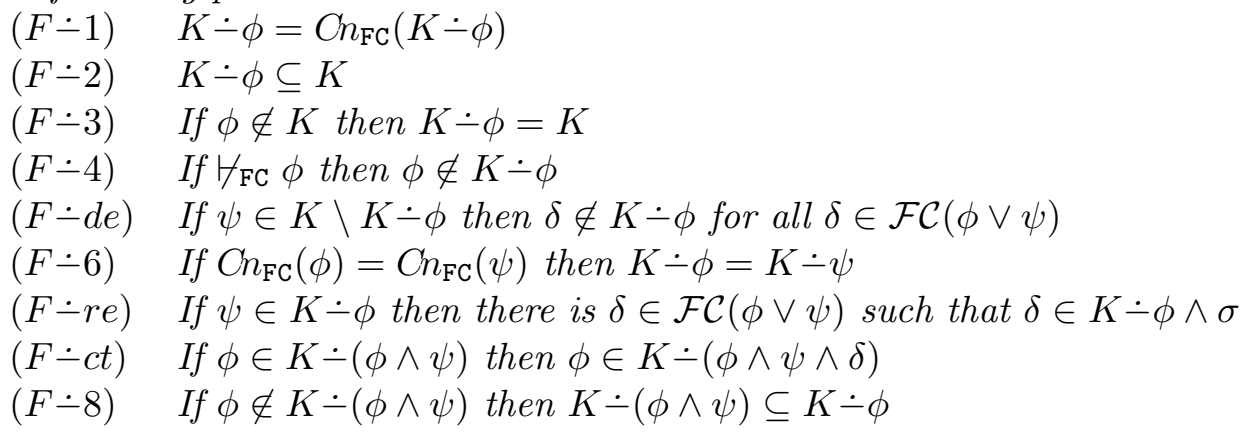

$(F \dot{-} 1)-(F \dot{-} 4),(F \dot{-} 6),(F \dot{-} c t)$, and $(F \dot{-} 8)$ are the $\left\langle\mathcal{L}_{\mathrm{FC}}, C n_{\mathrm{FC}}\right\rangle$ versions of $(K \dot{-} 1)-(K \dot{-} 4)$, $(K \dot{-} 6),(K \dot{-} c t)$, and $(K \dot{-} 8)$. Although not required for its characterisation, FC contraction also satisfies the $\left\langle\mathcal{L}_{\mathrm{FC}}, C n_{\mathrm{FC}}\right\rangle$ version of $(K \dot{-} 7)$.

7. "F" stands for fragment. 
Theorem 3. If a function - is an FC contraction function, then $\dot{-}$ satisfies the following postulate:

$(F \dot{-} 7) \quad K \dot{-} \phi \cap K \dot{-} \psi \subseteq K \dot{-}(\phi \wedge \psi)$

It follows from Theorems 2 and 3 that FC contraction complies with all the AGM contraction postulates except recovery. Furthermore, it complies with $(F \dot{-} d e),(F \dot{-} r e)$, and $(F \dot{-} c t)$ described below.

We suggest that the absence of recovery is not a weakness of FC contraction. Besides its controversy, satisfaction of recovery requires the property of AGM-compliance ${ }^{8}$ (Ribeiro, Wassermann, Flouris, \& Antoniou, 2013) of the underlying logic; and not all FC logics have this property. Additionally, we have $(F \dot{-} d e)$ to play the role of recovery. $(F \dot{-} d e)$ originates from the postulate of disjunctive elimination (Fermé, Krevneris, \& Reis, 2008):

$$
(K \dot{-} d e) \quad \text { If } \psi \in K \backslash K \dot{-} \phi \text { then } \phi \vee \psi \notin K \dot{-} \phi
$$

In its contrapositive form

$$
\text { If } \psi \in K \text { and } \phi \vee \psi \in K \dot{-} \phi \text { then } \psi \in K \dot{-} \phi
$$

$(K \dot{-} d e)$ is "a condition for a sentence $\psi$ 'to survive' the contraction process" (Fermé et al., $2008)$ (page 745$)$. So essentially $(K \dot{-} d e)$ specifies what should be retained after a contraction and in turn captures some minimal change properties of a contraction. $(K \dot{-} d e)$ is equivalent to recovery under propositional logic but unlike recovery it holds in our minimal $\operatorname{logic}\left\langle\mathcal{L}_{\mathrm{FC}}, C n_{\mathrm{FC}}\right\rangle .(K \dot{-} d e)$ refers to the disjunction $\phi \vee \psi$, which is the single closest FC approximation for $\phi \vee \psi$ under propositional logic. In a more general setting where disjunction is not fully supported, we have to refer to the disjunction by the notation $\mathcal{F C}(\phi \vee \psi)$, as in $(F \dot{-} d e)$.

As for $(F \dot{-} d e),(F \dot{-} r e)$ is neither a generalisation of an AGM contraction postulate nor a completely new postulate. Actually, it originates from a property of entrenchmentbased contraction. For entrenchment-based contraction, it follows from $(C \dot{-})$ that if $\psi$ is in $K \dot{-} \phi$, then $\phi \vee \psi$ is strictly more entrenched than $\phi$. Due to $(C \leq)$, this property of entrenchment-based contraction can be captured as follows:

$$
(K \dot{-} r e) \quad \text { If } \psi \in K \dot{-} \phi \text {, then } \phi \vee \psi \in K \dot{-} \phi \wedge(\phi \vee \psi)
$$

In the AGM setting, the property does not have to be postulated explicitly, as it follows from $(K \dot{-} 6)$ and $(K \dot{-1})$. Note that since $\phi \wedge(\phi \vee \psi)$ is logically equivalent to $\phi$, by $(K \dot{-} 6)$ we have $K \dot{-} \phi \wedge(\phi \vee \psi)=K \dot{-} \phi$. Then $\phi \vee \psi \in K \dot{-} \phi \wedge(\phi \vee \psi)$ follows from the fact that $\psi \in K \dot{-} \phi$ and $K \dot{-} \phi$ is logically closed. For FC contraction, it follows from $\left(C_{\mathrm{FC}} \dot{-}\right)$ that if $\psi$ is in $K \dot{-} \phi$, then there is an FC approximation of $\phi \vee \psi$ that is strictly more entrenched than $\phi$. Due to $(C \leq)$, this property is captured exactly by $(F \dot{-r e})$. And this time the postulate is not deducible from the others. ${ }^{9}$ Note that $(F \dot{-r e})$ generalises $(K \dot{-r e})$ in the same way as $(F \dot{-} d e)$ generalises $(K \dot{-} d e)$.

To further evaluate FC contraction, we apply it to propositional logic and its Horn fragment, since entrenchment-based contraction was originally defined for propositional

8. A logic $\langle\mathcal{L}, C n\rangle$ is AGM-compliant iff for all $K, A \subseteq \mathcal{L}$, where $A$ is finitely representable and $\operatorname{Cn}(\emptyset) \subset$ $C n(A) \subset C n(K)$, there is a $K^{\prime} \subseteq \mathcal{L}$ s.t. $\operatorname{Cn}\left(K^{\prime}\right) \subset C n(K)$ and $K^{\prime} \cup A=K$.

9. To show this, an example of a function is given in Appendix A such that it satisfies all characterising postulates of $\mathrm{FC}$ contraction and $(F \dot{-} 7)$ but violates $(F \dot{-} r e)$. 
logic and it has so far only been adapted to the Horn fragment (Zhuang \& Pagnucco, 2010, 2014). When the underlying logic is propositional logic, since it fully supports disjunction, the single closest FC approximation of $\phi \vee \psi$ is $\phi \vee \psi$ itself (up to logical equivalence) which means $(C \dot{-})$ and $\left(C_{\mathrm{FC}} \dot{-}\right)$ are equivalent. Therefore, when the underlying logic is propositional logic, FC contraction coincides with entrenchment-based contraction.

Proposition 2. Let the underlying logic be propositional logic. Then a function is an FC contraction function iff it is an entrenchment-based contraction function.

For the Horn fragment, the adapted version is called entrenchment-based Horn contraction and is based on the notion of Horn strengthenings. For any $\phi$ in $\mathcal{L}_{\mathrm{P}}$, its set of Horn strengthenings, denoted $\mathcal{H S}(\phi)$, consists of the logically weakest formulas in $\mathcal{L}_{\mathrm{H}}$ that entail $\phi$. The contraction outcome is determined by the condition $(H C \dot{-})$, which reformulates $(C \dot{-})$ with the notion of Horn strengthenings.

$$
\begin{gathered}
(H C \dot{-}) \quad \psi \in K \dot{-} \phi \text { iff } \psi \in K \text { and either there is } \delta \in \mathcal{H} \mathcal{S}(\phi \vee \psi) \text { such } \\
\text { that } \phi<\delta \text { or } \vdash_{\mathrm{P}} \phi .
\end{gathered}
$$

In $(H C \dot{-}), \leq$ is a binary relation over $\mathcal{L}_{\mathrm{H}}$ that satisfies the $\left\langle\mathcal{L}_{\mathrm{H}}, C n_{\mathrm{H}}\right\rangle$ version of $(E E 1)-$ (EE5); it is called Horn epistemic entrenchment. Clearly, an FC epistemic entrenchment is a Horn epistemic entrenchment under $\left\langle\mathcal{L}_{\mathrm{H}}, C n_{\mathrm{H}}\right\rangle$. To decide whether to retain $\psi$ when contracting $K$ by $\phi,(H C \dot{-})$ compares $\phi$ with the Horn strengthenings of $\phi \vee \psi$. By the definition of FC approximation, the set of Horn strengthenings of $\phi \vee \psi$ is exactly the set of closest $\mathrm{FC}$ approximations of $\phi \vee \psi$, which means $(H C \dot{-})$ and $\left(C_{\mathrm{FC}} \dot{-}\right)$ are equivalent. Therefore, when the underlying logic is the Horn fragment, FC contraction coincides with entrenchment-based Horn contraction.

Proposition 3. Let the underlying logic be the Horn fragment of propositional logic. A function is an FC contraction function iff it is an entrenchment-based Horn contraction function.

In summary, FC contraction complies with a set of postulates that are sufficient to characterise entrenchment-based contraction and all the characterising postulates for FC contraction originate from those for entrenchment-based contraction. Also FC contraction coincides with entrenchment-based contraction under propositional logic. These results suggest that FC contraction is no different from entrenchment-based contraction, only that the former is applicable to a much wider class of logics, and thus is a generalisation of the latter.

\section{FC Revision}

We have provided a generalisation of entrenchment-based contraction that is applicable to an arbitrary FC logic $\left\langle\mathcal{L}_{\mathrm{FC}}, C n_{\mathrm{FC}}\right\rangle$. In this section, we turn our attention to achieving the same for entrenchment-based revision.

The outcome of an entrenchment-based revision function is determined by an epistemic entrenchment via condition $(R *)$ which refers to the disjunction $\neg \phi \vee \psi$ and the negation $\neg \phi$. As neither $\neg \phi \vee \psi$ nor $\neg \phi$ are guaranteed to be in $\mathcal{L}_{\mathrm{FC}},(R *)$ in general cannot be applied in $\left\langle\mathcal{L}_{\mathrm{FC}}, C n_{\mathrm{FC}}\right\rangle$. As in reformulating condition $(C \dot{-})$, we give two methods in dealing 
with the inexpressible disjunction and negation, by working respectively with their FC approximations and cuts.

The first method gives rise to the following reformulated version of $(R *)$.

$\left(R_{\mathrm{FC}} *\right) \quad \psi \in K * \phi$ iff either there is $\delta \in \mathcal{F C}(\neg \phi \vee \psi)$ such that

$\sigma<\delta$ for all $\sigma \in \mathcal{F C}(\neg \phi)$ or $\vdash_{\mathrm{F}} \neg \phi$.

According to $\left(R_{\mathrm{FC}} *\right), \psi$ is in $K * \phi$ if and only if either there is an FC approximation of $\neg \phi \vee \psi$ that is strictly more entrenched than all FC approximations of $\neg \phi$, or $\neg \phi$ is a tautology. Note that if both $\neg \phi \vee \psi$ and $\neg \phi$ are in $\mathcal{L}_{\mathrm{FC}}$, then $\left(R_{\mathrm{FC}} *\right)$ is supposed to coincide with $(R *)$. For this, we require one (instead of all) FC approximations of $\neg \phi \vee \psi$ to be strictly more entrenched than all (instead of some) FC approximation of $\neg \phi$. Due to (EE2), we won't have the coincidence if it was "all" FC approximation of $\neg \phi \vee \psi$ or "some" FC approximation of $\neg \phi$.

Similar to $\left(C_{\mathrm{FC}} \dot{-}\right)$, it suffices to consider the closest FC approximations of $\neg \phi \vee \psi$ and $\neg \phi$ whenever they exist; that is given that $\mathcal{C F C}(\neg \phi \vee \psi)$ and $\mathcal{C F C}(\neg \phi)$ are non-empty, the condition obtained by replacing $\mathcal{F C}(\neg \phi \vee \psi)$ and $\mathcal{F C}(\neg \phi)$ with $\mathcal{C F C}(\neg \phi \vee \psi)$ and $\mathcal{C F C}(\neg \phi)$ respectively in $\left(R_{\mathrm{FC}} *\right)$ is equivalent to $\left(R_{\mathrm{FC}} *\right)$.

The second method is based on the cuts of $\neg \phi \vee \psi$ and $\neg \phi$. It is easy to see that, in the AGM setting, $\neg \phi<\neg \phi \vee \psi$ if and only if $c u t_{\leq}(\neg \phi \vee \psi) \subset c u t_{\leq}(\neg \phi)$. This gives rise to the following reformulated version of $(R *)$.

$$
\left(R_{\mathrm{FC}}^{c u t} *\right) \quad \psi \in K * \phi \text { iff either } c u t_{\leq}(\neg \phi \vee \psi) \subset c u t_{\leq}(\neg \phi) \text { or } \vdash_{\mathrm{F}} \neg \phi .
$$

Rather than looking for an FC approximation of $\neg \phi \vee \psi$ that is strictly more entrenched than all FC approximation of $\neg \phi$, with $\left(R_{\mathrm{FC}}^{c u t} \dot{-}\right)$, we check if the cut of $\neg \phi \vee \psi$ is a proper subset of that of $\neg \phi$. We can show that there exists one such FC approximation if and only if the set inclusion holds, which leads to the equivalence of $\left(R_{\mathrm{FC}} *\right)$ and $\left(R_{\mathrm{FC}}^{c u t} *\right)$.

Proposition 4. Let $\leq$ be an FC epistemic entrenchment for $K$. Then cut $_{\leq}(\neg \phi \vee \psi) \subset$ cut $_{\leq}(\neg \phi)$ iff there is $\delta \in \mathcal{F C}(\neg \phi \vee \psi)$ such that $\sigma<\delta$ for all $\sigma \in \mathcal{F C}(\neg \phi)$.

Formally, the generalisation of entrenchment-based revision for $\left\langle\mathcal{L}_{\mathrm{FC}}, C n_{\mathrm{FC}}\right\rangle$, called $F C$ revision, is defined as follows.

Definition 5. A function $*: 2^{\mathcal{L}_{\mathrm{FC}}} \times \mathcal{L}_{\mathrm{FC}} \mapsto 2^{\mathcal{L}_{\mathrm{FC}}}$ is an $F C$ revision function if $K * \phi$ is determined by an $F C$ epistemic entrenchment associated with $K$ via $\left(R_{\mathrm{FC}} *\right)$.

Due to the equivalence of $\left(R_{\mathrm{FC}} *\right)$ and $\left(R_{\mathrm{FC}}^{c u t} *\right), \mathrm{FC}$ contraction functions can be defined alternatively via $\left(R_{\mathrm{FC}}^{c u t} *\right)$. Both $\left(R_{\mathrm{FC}} *\right)$ and $\left(R_{\mathrm{FC}}^{c u t} *\right)$ are particularly useful in proving certain properties of FC revision, $\left(R_{\mathrm{FC}}^{c u t} *\right)$ avoids the computations of possibly infinitely many $\mathrm{FC}$ approximations, thus providing a viable option for implementing $\mathrm{FC}$ revision functions.

Next, we present some properties of FC revision. Given that the underlying FC logic $\left\langle\mathcal{L}_{\mathrm{FC}}, C n_{\mathrm{FC}}\right\rangle$ is capable of representing some tautologies, FC revision complies with all the AGM revision postulates. 
Theorem 4. Let $\mathcal{L}_{\mathrm{FC}}$ contains some tautologies. If a function $*$ is an $F C$ revision function, then it satisfies the following postulates:

$$
\begin{array}{ll}
(F * 1) & K * \phi=C n_{\mathrm{FC}}(K * \phi) \\
(F * 2) & K * \phi \subseteq K+\phi \\
(F * 3) & \text { If } K+\phi \text { is consistent then } K+\phi \subseteq K * \phi \\
(F * 4) & \phi \in K * \phi \\
(F * 5) & K * \phi=K_{\perp} \text { iff } \phi \text { is inconsistent } \\
(F * 6) & \text { If } C n_{\mathrm{FC}}(\phi)=C n_{\mathrm{FC}}(\psi) \text { then } K * \phi=K * \psi \\
(F * 7) & K *(\phi \wedge \psi) \subseteq(K * \phi)+\psi \\
(F * 8) & \text { If }(K * \phi)+\psi \text { is consistent then }(K * \phi)+\psi \subseteq K *(\phi \wedge \psi)
\end{array}
$$

$(F * 1)-(F * 8)$ are the $\left\langle\mathcal{L}_{\mathrm{FC}}, C n_{\mathrm{FC}}\right\rangle$ versions of $(K * 1)-(K * 8)$. The existence of tautologies in $\mathcal{L}_{\mathrm{FC}}$ is necessary for $\mathrm{FC}$ revision to comply with $(F * 4)$. This is because, if $\left\langle\mathcal{L}_{\mathrm{FC}}, C n_{\mathrm{FC}}\right\rangle$ cannot represent a tautology, then nothing prevents some $\sigma \in \mathcal{F C}(\neg \phi)$ being the most entrenched formula among $\mathcal{L}_{\mathrm{FC}}$ and in which case there is no $\delta \in \mathcal{F C}(\neg \phi \vee \psi)$ such that $\sigma<\delta$, meaning that $\phi \notin K * \phi$. FC revision still complies with the rest of the postulates in Theorem 4 if there is no tautology in $\mathcal{L}_{\mathrm{FC}}$.

As for FC contraction, we further evaluate $\mathrm{FC}$ revision by applying it to propositional logic and its Horn fragment. When the underlying logic is propositional, since it fully supports disjunction and negation, the single closest FC approximation of $\neg \phi \vee \psi$ is $\neg \phi \vee \psi$ itself (up to logical equivalence) and so is that of $\neg \phi$. Hence according to $(R *)$ and $\left(R_{\mathrm{FC}} *\right)$, when the underlying logic is propositional logic, FC revision coincides with entrenchmentbased revision

Proposition 5. Let the underlying logic be propositional logic. Then a function is an FC revision function iff it is an entrenchment-based revision function.

Entrenchment-based revision has not been adapted to the Horn fragment nor any other logic violating the AGM assumptions. But the model-based revision of Katsuno and Mendelzon (Katsuno \& Mendelzon, 1992) has been adapted to the Horn fragment by Delgrande and Peppas (2015) which we call model-based Horn revision. It makes sense to compare model-based Horn revision with the FC revision under the Horn fragment. Model-based Horn revision can be characterised by all the AGM revision postulates plus the following schema

(Acyc) If for $0 \leq i<n,\left(K * \phi_{i+1}\right)+\phi_{i}$ is consistent, and $\left(K * \phi_{0}\right)+\phi_{n}$ is consistent, then $\left(K * \phi_{n}\right)+\phi_{o}$ is consistent.

(Acyc) is implied by the AGM postulates under propositional logic, but is independent of the AGM postulates under the Horn fragment (Delgrande \& Peppas, 2015). By Theorem 4, FC revision satisfies all the AGM revision postulates, and we can prove that it also satisfies $(A c y c)$. Therefore, when the underlying logic is the Horn fragment, any FC revision function is a model-based revision function.

Proposition 6. Let the underlying logic be the Horn fragment of propositional logic. If a function is an FC revision function, then it is a model-based Horn revision function. 


\section{Revision via the Levi Identity}

Apart from classical constructions such as entrenchment-based revision, in the AGM setting, revision functions can also be constructed through contraction functions via the Levi identity. In this section we explore this approach in constructing revision functions that are applicable to an arbitrary FC logic $\left\langle\mathcal{L}_{\mathrm{FC}}, C n_{\mathrm{FC}}\right\rangle$. Also, we establish formal connections between the revision functions thus constructed and $\mathrm{FC}$ revision functions.

Following the Levi identity, a revision function $*$ would be constructed through an FC contraction function - as follows

$$
K * \phi=(K \dot{-} \neg \phi)+\phi .
$$

The obstacle here is that the negation $\neg \phi$ may not be in $\mathcal{L}_{\mathrm{FC}}$ and the FC contraction function - is not defined for formulas outside $\mathcal{L}_{\mathrm{FC}}$. Because of this, we instead consider the FC approximations of $\neg \phi$. In order to guarantee consistency after the expansion of $\phi$, all such approximations have to be removed from $K$. For this, before expanding by $\phi$, we contract by each FC approximation of $\neg \phi$, and then intersect the contraction outcomes. Moreover, we have to take care of the limiting case of $\mathcal{F C}(\neg \phi)=\emptyset$. In this case, $K$ is guaranteed to be consistent with $\phi$, hence the contraction step can be avoided. We call the revision functions constructed in the above manner contraction generated revision functions.

Definition 6. A function $*: 2^{\mathcal{L}_{\mathrm{FC}}} \times \mathcal{L}_{\mathrm{FC}} \mapsto 2^{\mathcal{L}_{\mathrm{FC}}}$ is a contraction generated revision function iff

$$
K * \phi= \begin{cases}\bigcap_{\sigma \in \mathcal{F} \mathcal{C}(\neg \phi)}(K \dot{-} \sigma)+\phi & \text { if } \mathcal{F C}(\neg \phi) \neq \emptyset \\ K+\phi & \text { otherwise }\end{cases}
$$

where - is an FC contraction function.

The belief revision community generally understands a revision, defined via the Levi identity, as a contraction step followed by an expansion step. This, however, is a simplification of Levi's original idea (Levi, 1991) as expressed by his Commensurability Thesis (page 65). This thesis essentially states that one can get from one state of belief to another through a sequence of expansions and contractions. Therefore, the way we obtain contraction generated revision by performing multiple contractions followed by an expansion is in accordance with Levi's original idea on the nature of the revision operation.

Given that the underlying FC logic $\left\langle\mathcal{L}_{\mathrm{FC}}, C n_{\mathrm{FC}}\right\rangle$ is capable of representing some tautologies, we can show that, under a finiteness condition, contraction generated revision complies with all the AGM revision postulates.

Theorem 5. Let $\mathcal{L}_{\mathrm{FC}}$ contains some tautologies and let $\mathcal{F C}(\neg \phi)$ be finite (up to logical equivalence) for all $\phi \in \mathcal{L}_{\mathrm{FC}}$. If a function $*$ is a contraction generated revision function, then it satisfies $(F * 1)-(F * 8)$.

As for Theorem 4, the existence of tautologies is necessary for proving the satisfaction of $(F * 4)$. For the finiteness condition, it guarantees the existence of a most entrenched formula in $\mathcal{F C}(\neg \phi)$, which is required in proving the satisfaction of $(F * 8)$. It is also required in proving the equivalence of $\mathrm{FC}$ revision and contraction generated revision. 
Theorem 6. Let $\mathcal{F C}(\neg \phi)$ be finite (up to logical equivalence) for all $\phi \in \mathcal{L}_{\mathrm{FC}}$. A function is an $F C$ revision function iff it is a contraction generated revision function.

Although the compliance with AGM revision postulates and the equivalence with FC revision are desirable, the fact that both results rely on a finiteness condition makes them less appealing. In the remainder of this section, we explore the notion of revision equivalence (Makinson, 1987) which leads to two conceptually and computationally simpler constructions for revision functions. More importantly, the two constructions do not require any finiteness condition for the equivalence with FC revision and hence the compliance with AGM revision postulates.

In the AGM setting, a revision function constructed through an AGM contraction function can also be constructed through a withdrawal function (Makinson, 1987). A withdrawal function refers to a contraction function that does not necessarily satisfy the recovery postulate, and often has a simpler construction than AGM contraction functions. The withdrawal version of entrenchment-based contraction called entrenchment-based withdrawal (Rott, 1991; Rott \& Pagnucco, 1999) is based on the following condition in the same way as entrenchment-based revision is based on $(R *)$.

$(W \dot{\bullet}) \psi \in K \dot{-} \phi$ iff $\psi \in K$ and either $\phi<\psi$ or $\vdash \phi$.

According to $(W \dot{-}), \psi$ is in $K \dot{-} \phi$ if and only if it is a formula of $K$ and it is strictly more entrenched than $\phi$ or $\phi$ is a tautology. In other words, whenever $\phi$ is not a tautology, $K \dot{-} \phi$ consists of all formulas that are strictly more entrenched than $\phi$, that is

$$
K \dot{-} \phi=\mathrm{cut}_{\leq}(\phi)
$$

where $\leq$ is an epistemic entrenchment associated with $K$. Entrenchment-based withdrawal and entrenchment-based contraction are revision equivalence, that is for each entrenchmentbased contraction function, there is an entrenchment-based withdrawal function such that the revision function it generates via the Levi identity is identical to the one generated by the entrenchment-based contraction function and vice versa (Rott, 1991; Rott \& Pagnucco, 1999).

Entrenchment-based withdrawal can be easily generalised to $\mathrm{FC}$ logics. First, $(W \dot{-})$ can be reformulated as follows with $\left\langle\mathcal{L}_{\mathrm{FC}}, C n_{\mathrm{FC}}\right\rangle$ as the underlying logic.

$$
\left(W_{\mathrm{FC}} \dot{-}\right) \psi \in K \dot{-} \phi \text { iff } \psi \in K \text { and either } \phi<\psi \text { or } \vdash_{\mathrm{F}} \phi \text {. }
$$

Then an FC logic version of entrenchment-based withdrawal which we call $F C$ withdrawal function can be defined as follows.

Definition 7. A function $\dot{-}: 2^{\mathcal{L}_{\mathrm{FC}}} \times \mathcal{L}_{\mathrm{FC}} \mapsto 2^{\mathcal{L}_{\mathrm{FC}}}$ is an $F C$ withdrawal function if $K \dot{-} \phi$ is determined by an $F C$ epistemic entrenchment associated with $K$ via $\left(W_{\mathrm{FC}} \dot{-}\right)$.

The construction of FC withdrawal is computationally simpler than that of FC contraction since it avoids some of the intricate computations for FC contraction including the computation of $c u t_{\leq}(\phi)$, and the determination of whether $c u t_{\leq}(\phi) \vdash_{\mathrm{F}} \phi \vee \psi$.

As for the construction of contraction generated revision, we can construct a revision function through an FC withdrawal function, which we call withdrawal generated revision function. 
Definition 8. A function $*: 2^{\mathcal{L}_{\mathrm{FC}}} \times \mathcal{L}_{\mathrm{FC}} \mapsto 2^{\mathcal{L}_{\mathrm{FC}}}$ is a withdrawal generated revision function iff

$$
K * \phi= \begin{cases}\bigcap_{\sigma \in \mathcal{F} \mathcal{C}(\neg \phi)}(K \dot{-} \sigma)+\phi & \text { if } \mathcal{F} \mathcal{C}(\neg \phi) \neq \emptyset \\ K+\phi & \text { otherwise }\end{cases}
$$

where - is an FC withdrawal function.

It turns out that, under the same finiteness condition as in Theorem 5 and 6 , contraction generated revision and withdrawal generated revision are equivalent which implies the revision equivalence of FC contraction and FC withdrawal (under the finiteness condition).

Theorem 7. Let $\mathcal{F C}(\neg \phi)$ be finite (up to logical equivalence) for all $\phi \in \mathcal{L}_{\mathrm{FC}}$. A function is a contraction generated revision function iff it is a withdrawal generated revision function.

Again the finiteness condition guarantees the existence of a most entrenched formula in $\mathcal{F C}(\neg \phi)$, which is required in deducing the entrenchment relation (i.e., $\phi<\psi)$ in $\left(W_{\mathrm{FC}} \dot{-}\right)$ from the corresponding one (i.e., $\phi<\sigma$ for all $\sigma \in \mathcal{F C}(\neg \phi))$ in $\left(C_{\mathrm{FC}} \dot{-}\right)$ and hence the proof of Theorem 7. It follows from Theorem 6 and 7 that withdrawal generated revision and FC revision are equivalent under this finiteness condition. In fact, we can prove a stronger result that the equivalence holds even when the finiteness condition does not.

Theorem 8. A function is an FC revision function iff it is a withdrawal generated revision function.

It is evident that FC withdrawal is a better alternative than FC contraction for constructing revision functions via the Levi identity. Actually, we can come up with another better alternative. Due to the fact that an entrenchment-based withdrawal function $\dot{-}$ is such that $K \dot{-} \phi=c u t_{\leq}(\phi)$ whenever $\phi$ is non-tautological, and the revision equivalence of entrenchment-based withdrawal and contraction, an AGM revision revision function $*$ can be constructed as

$$
K * \phi=\operatorname{cut}_{\leq}(\neg \phi)+\phi
$$

where $\leq$ is an epistemic entrenchment associated with $K$. Since $c u t_{\leq}(\neg \phi)$ is well-defined in any $\mathrm{FC}$ logic, we can constructed a revision function in the same manner for $\left\langle\mathcal{L}_{\mathrm{FC}}, C n_{\mathrm{FC}}\right\rangle$, which we call cut generated revision function.

Definition 9. A function $*: 2^{\mathcal{L}_{\mathrm{FC}}} \times \mathcal{L}_{\mathrm{FC}} \mapsto 2^{\mathcal{L}_{\mathrm{FC}}}$ is a cut generated revision function iff

$$
K * \phi=\operatorname{cut}_{\leq}(\neg \phi)+\phi
$$

where $\leq i s$ an epistemic entrenchment associated with $K$.

As for withdrawal generated revision, we can show the equivalence of cut generated revision and $\mathrm{FC}$ revision and hence the revision equivalence of withdrawal and cut generated revision.

Theorem 9. A function is an FC revision function iff it is a cut generated revision function. 
Note that $\mathcal{F C}(\neg \phi)$ could be an infinite set, in which case it requires infinitely many contraction operations to remove each element in $\mathcal{F C}(\neg \phi)$ individually from a belief set. For this, both withdrawal and contraction generated revision may involve an infinite number of contraction operations. Clearly, this is not an issue for cut generated revision and in this sense, it is superior to both withdrawal and contraction generated revision. Cut generated revision is also superior to FC revision, as it only needs to obtain the cut of $\neg \phi$ for the revision by $\phi$, whereas FC revision needs to obtain the cut of $\neg \phi$ and that of $\neg \phi \vee \psi$ for every $\psi$ in $K$.

In summary, for revision under FC logics, we have introduced FC revision which generalises entrenchment-based revision alone with contraction, withdrawal, and cut generated revision which generalises the construction of revision via the Levi identity. Due to their equivalence, for a particular application scenario and underlying logic, we are free to use any of them that is most suitable. Having said that, we argue that cut generated revision is the better option for most real-world applications because of its simplicity.

\section{Related Work}

Most works on generalising AGM contraction and revision have targeted the Horn fragment of propositional logic and description logics. The initial works for the Horn fragment focused on contraction. We have shown that FC contraction when applied to the Horn fragment coincides with entrenchment-based Horn contraction. Other than entrenchment-based Horn contraction, there have been two main approaches in generalising partial meet contraction (Alchourrón et al., 1985) to the Horn fragment, one by Delgrande and Wassermann (2013) and one by Booth et al. (2011). The two approaches differ in the techniques used for adapting the notion of remainder sets to the Horn fragment. Zhuang and Pangucco (2011) further studied the approach of Delgrande and Wassermann, which resulted in a version of transitively relational partial meet contraction (Alchourrón et al., 1985) for the Horn fragment. They have also given an equivalent model-based approach (Zhuang \& Pagnucco, 2012).

Apart from contraction, Delgrande and Peppas (2015) studied the operation of revision for the Horn fragment. This work has been extended to arbitrary logics (Delgrande et al., 2018), but the fundamental ideas are the same as what follows. By following Katsuno and Mendelzon (1992), they defined model-based Horn revision such that the revision outcome is determined by faithful pre-orders of propositional interpretations. In addition to the AGM revision postulates, the schema (Acyc) is required to characterise their revision functions. Pre-orders are by definition acyclic; however, under the Horn fragment some revision functions defined in the manner of Katsuno and Mendelzon (1992) but through cyclic orderings of interpretations also comply with the AGM revision postulates. (Acyc) rules out such functions by enforcing transitivity on the determining orderings. We have shown that under the Horn fragment any FC revision function is a model-based Horn revision function.

AGM contraction and revision are inter-definable via the Levi and Harper identities. Zhuang, Pagnucco, and Zhang $(2013,2017)$ investigated the inter-definability of contraction and revision when the underlying logic is the Horn fragment. Since the Horn fragment does not fully support negation, in defining revision from contraction they were confronted with a similar obstacle as we were in defining revision from FC contraction. Our technique of 
using FC approximations to get around the obstacle is a generalisation of their technique of using Horn strengthening. They identified a sufficient condition for a contraction function to generate a revision function that complies with all the AGM revision postulates, which they call plausible revision functions. However, they did not give a conclusive answer regarding whether all entrenchment-based Horn contraction functions generate a plausible revision function. Our results on contraction generated revision confirm this.

For the Horn fragment, we also note that Wu, Zhang, and Zhang (2011) studied contraction within the framework of relevance-based belief change; Langlois, Sloan, Szörényi, and Turán (2008) studied revision by characterising the existence of a complement of a Horn consequence; and Adaricheva, Sloan, and Szörényi (2012) provided some complexity results on contraction.

Going beyond the Horn fragment, Creignou et al. (2014) studied revision for the Krom, affine, and the dual Horn fragment of propositional logic. Their aim, however, is different from ours. They focus on refining classical revision operators to guarantee the revision outcome is always expressible in the background propositional fragment and in doing so preserve as much as possible the original behaviours of the classical revision operators.

In contrast to the works for the Horn fragment, most works targeting description logics focus on belief base contraction and revision (Hansson, 1999). As well, they do not assume any explicit preference relation, for example an epistemic entrenchment or a pre-order of interpretations, in determining the contraction and revision outcome (e.g., Qi \& Du, 2009; Qi et al., 2008; Zhuang et al., 2014; Wang et al., 2015b; Zhuang et al., 2016). One exception is the work by Wang et al. (2015a) which applied Satoh's operator (Satoh, 1988) to DLLite (Calvanese et al., 2007). As is intrinsic with Satoh's operator, the approach does not comply with all the AGM revision postulates.

Finally, we note that Ribeiro et al. (2013) aims at generalising AGM contraction to both the propositional fragments and description logics. They identify sufficient conditions on the background logic for the possibility of defining a contraction function that satisfies the postulate of recovery and relevance (Hansson, 1991). According to their results, FC logic, in general, does not allow contraction functions to be defined that complies with recovery.

\section{Conclusion and Future Work}

In this paper, some core construction methods for AGM contraction and revision functions are generalised to fragments of first-order logic that support conjunction and allow only closed formulas. The generalisation is first carried out for entrenchment-based contraction, with the generalised version called FC contraction. The generality is achieved by basing FC contraction on the notion of FC approximations or on that of cut, which impose no requirement on the expressiveness of the underlying logic. Arguably since the characterising postulates for $\mathrm{FC}$ contraction are independent of the specific underlying logic, they are more suitable than the AGM contraction postulates for capturing the rationale behind a contraction operation. The generalisation is subsequently carried out for entrenchmentbased revision with the generalised version called FC revision, which in turn comply with all the AGM revision postulates. Due to the more involved construction steps we have not been able to give a representation theorem for FC revision. Finally, we generalised the construction of revision functions via the Levi identity and explored the notion of 
revision equivalence. This leads to three methods for generating revision functions, namely contraction, withdrawal, and cut generated revision. While being equivalent to FC revision, both withdrawal and cut generated revision have a simpler construction.

For future work, we will look for the set of postulates that are sufficient to characterise FC revision. A short-cut to approach this is to characterise instead cut generated revision, as cut generated revision is conceptually more straightforward than FC revision yet is equivalent to $\mathrm{FC}$ revision.

\section{Acknowledgments}

This work was supported by Australian Research Council (ARC) under DP130102302. Zhiqiang Zhuang was working at the Institute for Integrated and Intelligent Systems of Griffith University while conducting this work. James Delgrande gratefully acknowledges financial support from the Natural Sciences and Engineering Research Council of Canada.

\section{Appendix A}

In this appendix, we give some auxiliary lemmas that are required for proving the main results. We also give two examples. The first one shows that there are FC logics and formulas $\phi$ such that the set of closest FC approximations of $\phi$ is empty and the set of FC approximations of $\phi$ is infinite; and the second one shows that $(F \dot{-} r e)$ is not deducible from the other characterising postulates of $\mathrm{FC}$ contraction and $(F \dot{-} 7)$. Recall that unless stated otherwise, we work with the indefinite $\mathrm{FC}$ logic denoted as $\left\langle\mathcal{L}_{\mathrm{FC}}, C n_{\mathrm{FC}}\right\rangle$.

Lemma 1. Let $\leq$ be an FC epistemic entrenchment. Then $\leq$ satisfies:

1. $\phi \leq \psi$ or $\psi \leq \phi$

2. If $\psi \wedge \sigma \leq \phi$, then $\psi \leq \phi$ or $\sigma \leq \phi$

3. $\phi<\psi$ iff $\phi \wedge \psi<\psi$

4. If $\sigma \leq \phi$ and $\sigma \leq \psi$, then $\sigma \leq \phi \wedge \psi$

5. If $\sigma<\phi$ and $\sigma<\psi$, then $\sigma<\phi \wedge \psi$

6. If $\phi \leq \psi$, then $\phi \leq \phi \wedge \psi$

7. If $C n_{\mathrm{FC}}(\phi)=C n_{\mathrm{FC}}(\psi)$ then $\phi \leq \psi$ and $\psi \leq \phi$

Proof. Follows immediately from the definition of FC epistemic entrenchments.

Lemma 2. Let $\Delta \cup\{\phi\} \subseteq \mathcal{L}_{\mathrm{FC}}$. Then $\Delta \cup\{\phi\} \vdash_{\mathrm{F}} \psi$ iff there is $\delta \in \mathcal{F C}(\neg \phi \vee \psi)$ such that $\Delta \vdash_{\mathrm{FC}} \delta$.

Proof. $\Rightarrow$ : Suppose $\Delta \cup\{\phi\} \vdash_{\mathrm{F}} \psi$. We have by the deduction theorem that $\Delta \vdash_{\mathrm{F}} \phi \rightarrow \psi$. Then we have by the compactness theorem that there is a finite subset $\left\{\delta_{1}, \ldots, \delta_{n}\right\}$ of $\Delta$ such that $\left\{\delta_{1}, \ldots, \delta_{n}\right\} \vdash_{\mathrm{F}} \phi \rightarrow \psi$. Thus $\Delta \vdash_{\mathrm{FC}} \delta_{1} \wedge \cdots \wedge \delta_{n}$ and $\delta_{1} \wedge \cdots \wedge \delta_{n} \vdash_{\mathrm{F}} \phi \rightarrow \psi$. Then it follows from $\delta_{1} \wedge \cdots \wedge \delta_{n} \in \mathcal{L}_{\mathrm{FC}}$ that $\delta_{1} \wedge \cdots \wedge \delta_{n} \in \mathcal{F C}(\phi \rightarrow \psi)$. That is $\delta_{1} \wedge \cdots \wedge \delta_{n} \in$ $\mathcal{F C}(\neg \phi \vee \psi)$ as $C n_{\mathrm{F}}(\phi \rightarrow \psi)=C n_{\mathrm{F}}(\neg \phi \vee \psi)$. 
$\Leftarrow$ : Suppose there is $\delta \in \mathcal{F C}(\neg \phi \vee \psi)$ such that $\Delta \vdash_{\mathrm{FC}} \delta$. Then we have $\delta \vdash_{\mathrm{F}} \neg \phi \vee \psi$, which implies $\Delta \vdash_{\mathrm{F}} \neg \phi \vee \psi$. Thus $\Delta \cup\{\phi\} \vdash_{\mathrm{F}} \psi$.

Example 1. ${ }^{10}$ Let $\mathcal{L}_{\mathrm{FC}}$ consist of all ground formulas that can be constructed from the unary predicate $P$, the unary function $f$, and the constant $c$. Then $\exists x P(x) \notin \mathcal{L}_{\mathrm{FC}}$. Let's try to work out the closest $F C$ approximations of $\exists x P(x)$. For any $\sigma \in \mathcal{L}_{\mathrm{FC}}$ such that $\sigma \vdash_{\mathrm{F}} \exists x P(x)$ we can construct an infinite chain of formulas each of which entails $\exists x P(x)$ :

$$
\begin{aligned}
& \sigma_{1}=\sigma \vee P(f(c)) \\
& \sigma_{2}=\sigma \vee P(f(c)) \vee P(f(f(c))) \\
& \sigma_{3}=\sigma \vee P(f(c)) \vee P(f(f(c))) \vee P(f(f(f(c))))
\end{aligned}
$$

Since all $\sigma_{i}$ are in $\mathcal{L}_{\mathrm{FC}}$ and $\sigma_{i+1}$ is logically weaker than $\sigma_{i}$, there is no weakest formula in the chain which implies there exists no closest $F C$ approximation of $\exists x P(x)$ with respect to $\left\langle\mathcal{L}_{\mathrm{FC}}, C n_{\mathrm{FC}}\right\rangle$, that is $\mathcal{C F C}\left(\exists x P(x), \mathcal{L}_{\mathrm{FC}}\right)=\emptyset$. Also, due to the infinity of the chain and the fact that all $\sigma_{i}$ entail $\exists x P(x)$, the set $\mathcal{F C}\left(\exists x P(x), \mathcal{L}_{\mathrm{FC}}\right)$ is infinite.

Example 2. Let the underlying logic be $\left\langle\mathcal{L}_{\mathrm{H}}, C n_{\mathrm{H}}\right\rangle$ such that the language $\mathcal{L}_{\mathrm{H}}$ is over the set of atoms $\{p, q, r\}$. We denote an interpretation as a vector of $1 s$ and $0 s$. For instance an interpretation 101 indicates $p$ is assigned true, $q$ is assigned false, and $r$ is assigned true. We denote the set of models of a formula $\phi$ and a set of formulas $S$ as $|\phi|$ and $|S|$ respectively.

We consider the model-based Horn contraction function defined in (Zhuang \& Pagnucco, 2012). Formally, a function $-: 2^{\mathcal{L}_{\mathrm{H}}} \times \mathcal{L}_{\mathrm{H}} \mapsto 2^{\mathcal{L}_{\mathrm{H}}}$ is a model-based Horn contraction function iff

$$
K \dot{-} \phi=\mathcal{T}_{\mathrm{H}}(|K| \cup \min (|\neg \phi|, \preceq)) .
$$

where $\preceq$ is a total and faithful pre-order over all propositional interpretations; $\min (|\neg \phi|, \preceq$ )$=\{\mu \in|\neg \phi|$ : there is no $\nu \in|\neg \phi|$ such that $\nu \prec \mu\}$; and $\mathcal{T}_{\mathrm{H}}(|K| \cup \min (|\neg \phi|, \preceq))=\{\psi \in$ $\left.\mathcal{L}_{\mathrm{H}}:|K| \cup \min (|\neg \phi|, \preceq) \subseteq|\psi|\right\}$.

It is shown that a model-based Horn contraction function complies with $(F-1)-(F-4)$, $(F \dot{-} d e),(F-6)-(F \dot{-} 8)$, and $(F \dot{-} c t)$ (Zhuang 85 Pagnucco, 2011; Zhuang et al., 2013, 2017). We are going to show that it violates $(F \dot{-} r e)$.

Let $K$ be $C n_{\mathrm{H}}((\neg p \vee q) \wedge(\neg p \vee r))$ and the pre-order $\preceq$ for $\dot{-}$ be

$$
\text { all other interpretations } \prec 101 \prec 110 \prec 100
$$

10. This example is a simplification of the one given by an anonymous reviewer for when the paper was submitted to Artificial Intelligence Journal. 
Then we have

$$
\begin{aligned}
|\neg p \vee q| & =|K| \cup\{110\}, \\
|\neg(\neg p \vee r)| & =\{110,100\}, \\
|\neg((\neg p \vee r) \wedge(\neg p \vee q))| & =\{101,100,110\}, \\
|K \dot{-}(\neg p \vee r)| & =|K| \cup\{110\}, \text { and } \\
|K \dot{-}(\neg p \vee r) \wedge(\neg p \vee q)| & =|K| \cup\{101\} .
\end{aligned}
$$

Thus

$$
\begin{aligned}
& \neg p \vee q \in K \dot{-}(\neg p \vee r) \text { and } \\
& \neg p \vee q \notin K \dot{-}-(\neg p \vee r) \wedge(\neg p \vee q)
\end{aligned}
$$

Moreover, it follows from $(F \dot{-} 4)$ and $(F \dot{-} 6)$ that $\neg p \vee r \notin K \dot{-}(\neg p \vee r) \wedge(\neg p \vee r)=K \dot{-}(\neg p \vee r)$; $\neg p \notin K \dot{-}(\neg p \vee r) \wedge \neg p=K \dot{-} \neg p$; and $r \notin K \dot{-}(\neg p \vee r) \wedge r=K \dot{-} r$. Finally, since $q \notin K$, it follows from $(F-2)$ that $q \notin K \dot{-}(\neg p \vee r) \wedge q$.

Note that $\mathcal{F C}((\neg p \vee r) \vee(\neg p \vee q))=\{\perp, \neg p, q, r, \neg p \vee q, \neg p \vee r\}$. If we let $\phi$ be $\neg p \vee r$ and $\psi$ be $\neg p \vee q$, then it is clear that $(F \dot{-} r e)$ is violated.

\section{Appendix B}

In this appendix we show the proofs of the main results in this paper.

\section{Proof of Proposition 1}

$\Rightarrow$ : Suppose cut $_{\leq}(\phi) \vdash_{\mathrm{F}} \phi \vee \psi$. Then there is $\delta \in \mathcal{F C}(\phi \vee \psi)$ such that $\delta \in$ cut $_{\leq}(\phi)$. Since $\phi \notin$ cut $_{\leq}(\phi)$, it follows from the definition of cut that $\phi<\delta$.

$\Leftarrow$ : Suppose there is $\delta \in \mathcal{F C}(\phi \vee \psi)$ such that $\phi<\delta$. It then follows from (EE2) that $\{\sigma \in K \mid \delta \leq \sigma\} \nvdash_{\mathrm{FC}} \phi$ which implies $\delta \in c u t_{\leq}(\phi)$. Then since $\delta \vdash_{\mathrm{F}} \phi \vee \psi$, we have cut $_{\leq}(\phi) \vdash_{\mathrm{F}} \phi \vee \psi$.

\section{Proof of Theorem 1}

$\Rightarrow$ : Suppose $\phi \leq \psi$ and $\phi \in K \dot{-} \phi \wedge \psi$. We need to show $\vdash_{\mathrm{FC}} \phi \wedge \psi$. Since $\phi \in K \dot{-} \phi \wedge \psi$ we have by $\left(C_{\mathrm{FC}} \dot{-}\right)$ that either $\vdash_{\mathrm{FC}} \phi \wedge \psi$ or there is $\sigma \in \mathcal{F C}(\phi \vee(\phi \wedge \psi))$ such that $\phi \wedge \psi<\sigma$. If it is the latter case, then we have $\sigma \vdash_{\mathrm{F}} \phi \vee(\phi \wedge \psi)$. Since $C n_{\mathrm{F}}(\phi \vee(\phi \wedge \psi))=\operatorname{Cn}_{\mathrm{F}}(\phi)$, we have $\sigma \vdash_{\mathrm{FC}} \phi$ which implies by $(E E 2)$ that $\sigma \leq \phi$. Since $\phi \leq \psi$, we have by Lemma 1 (part $6)$ that $\phi \leq \phi \wedge \psi$. Then it follows from $(E E 1)$ and $\sigma \leq \phi$ that $\sigma \leq \phi \wedge \psi$ which implies $\phi \wedge \psi \nless \sigma$, a contradiction. Thus the former case must hold.

$\Leftarrow$ : If $\vdash_{\mathrm{FC}} \phi \wedge \psi$, then both $\phi$ and $\psi$ are tautologies. Thus $\phi \leq \psi$ follows from (EE5). So suppose $\nvdash_{\text {FC }} \phi \wedge \psi$ and $\phi \notin K \dot{-} \phi \wedge \psi$. We need to show $\phi \leq \psi$. Since $\phi \notin K \dot{-} \phi \wedge \psi$, we have by $\left(C_{\mathrm{FC}} \dot{-}\right)$ that either $\phi \notin K$ or $\phi \wedge \psi \nless \sigma$ for all $\sigma \in \mathcal{F C}(\phi \vee(\phi \wedge \psi))$. For the former case, $\phi \notin K$ gives us $\phi \leq \psi$ as required by $(E E 4)$. Since $C n_{\mathrm{F}}(\phi \vee(\phi \wedge \psi))=\operatorname{Cn}_{\mathrm{F}}(\phi)$, we have $\phi \in \mathcal{F C}(\phi \vee(\phi \wedge \psi))$. Thus for the latter case, we have $\phi \wedge \psi \nless \phi$ which means $\phi \leq \phi \wedge \psi$. Then since $(E E 2)$ and $\phi \wedge \psi \vdash_{\mathrm{FC}} \psi$ give us $\phi \wedge \psi \leq \psi$, we have by (EE1) that $\phi \leq \psi$. 


\section{Proof of Theorem 2}

$\Rightarrow$ : Suppose the function - is an FC contraction function and the FC epistemic entrenchment associated with $K$ is $\leq$. We need to show $\dot{-}$ satisfies $(F \dot{-} 1)-(F \dot{-} 4),(F \dot{-} d e)$, $(F \dot{-} 6),(F \dot{-} r e),(F \dot{-} c t)$, and $(F \dot{-} 8)$. Note that Theorem 1 assures that $\dot{-}$ satisfies $(C \leq)$.

$(F \dot{-1}):$ If $\vdash_{\mathrm{FC}} \phi$, then it follows from $\left(C_{\mathrm{FC}} \dot{\dot{ }}\right)$ that $K \dot{-} \phi=K$. Since $K=\operatorname{Cn}_{\mathrm{FC}}(K)$, we have $K \dot{-} \phi=C n_{\mathrm{FC}}(K \dot{-} \phi)$. So suppose $\nvdash_{\mathrm{FC}} \phi$ and $\psi \in C n_{\mathrm{FC}}(K \dot{-} \phi)$. We need to show $\psi \in K \dot{-} \phi$. By $\left(C_{\mathrm{FC}} \dot{-}\right)$ it suffices to show $\psi \in K$ and there is $\sigma \in \mathcal{F C}(\phi \vee \psi)$ such that $\phi<\sigma$. We have two cases:

Case $1, \nvdash_{\mathrm{FC}} \psi$ : Since $\psi \in C n_{\mathrm{FC}}(K \dot{-} \phi)$, by the compactness of $\left\langle\mathcal{L}_{\mathrm{FC}}, C n_{\mathrm{FC}}\right\rangle$ (follows from the compactness theorem), there is a finite subset $\left\{\delta_{1}, \ldots, \delta_{n}\right\}$ of $K \dot{-} \phi$ such that $\delta_{1} \wedge \cdots \wedge \delta_{n} \vdash_{\mathrm{FC}} \psi$. Since $\left\{\delta_{1}, \ldots, \delta_{n}\right\} \subseteq K \dot{-} \phi,(F \dot{-} 2)$ implies $\left\{\delta_{1}, \ldots, \delta_{n}\right\} \subseteq K$. It follows from $\left\{\delta_{1}, \ldots, \delta_{n}\right\} \subseteq K$ and $\delta_{1} \wedge \cdots \wedge \delta_{n} \vdash_{\mathrm{FC}} \psi$ that $K \vdash_{\mathrm{FC}} \psi$. It follows from $K \vdash_{\mathrm{FC}} \psi$ and $K=C n_{\mathrm{FC}}(K)$ that $\psi \in K$. Since $\left\{\delta_{1}, \ldots, \delta_{n}\right\} \subseteq K \dot{-} \phi$, it follows from $\left(C_{\mathrm{FC}} \dot{-}\right)$ that there is $\sigma_{i} \in \mathcal{F C}\left(\phi \vee \delta_{i}\right)$ such that $\phi<\sigma_{i}$ for $1 \leqslant i \leqslant n$. By Definition 2 , this means $\sigma_{i} \in \mathcal{L}_{\mathrm{FC}}$ and $\sigma_{i} \vdash_{\mathrm{F}} \phi \vee \delta_{i}$ for $1 \leqslant i \leqslant n$ which implies $\sigma_{1} \wedge \cdots \wedge \sigma_{n} \vdash_{\mathrm{F}} \phi \vee\left(\delta_{1} \wedge \cdots \wedge \delta_{n}\right)$. Thus $\sigma_{1} \wedge \cdots \wedge \sigma_{n} \vdash_{\mathrm{F}} \phi \vee \psi$. It follows from $\sigma_{1} \wedge \cdots \wedge \sigma_{n} \in \mathcal{L}_{\mathrm{FC}}$ and $\sigma_{1} \wedge \cdots \wedge \sigma_{n} \vdash_{\mathrm{F}} \phi \vee \psi$ that $\sigma_{1} \wedge \cdots \wedge \sigma_{n} \in \mathcal{F C}(\phi \vee \psi)$. Since $\phi<\sigma_{i}$ for $1 \leq i \leq n$, we have by Lemma 1 (part 5 ) that $\phi<\sigma_{1} \wedge \cdots \wedge \sigma_{n}$.

Case $2, \vdash_{\mathrm{FC}} \psi$ : Since $(F \dot{-} 2)$ gives $K \dot{-} \phi \subseteq K$, it follows from $\psi \in K \dot{-} \phi$ that $\psi \in K$. Also $\vdash_{\mathrm{FC}} \psi$ implies $\vdash_{\mathrm{FC}} \phi \vee \psi$. Then by Definition $2, \psi \in \mathcal{F C}(\phi \vee \psi)$. Finally, it follows from $\nvdash_{\mathrm{FC}} \phi$ and $(E E 5)$ that $\phi<\psi$.

$(F \dot{-} 2)$ : Follows directly from $\left(C_{\mathrm{FC}} \dot{-}\right)$.

$(F \dot{-} 3)$ : Suppose $\phi \notin K . K \dot{-} \phi \subseteq K$ is given by $(F \dot{-} 2)$. Suppose $\psi \in K$. We are going to show $\psi \in K \dot{-} \phi$. It follows from $\psi \vdash_{\mathrm{F}} \phi \vee \psi$ and $\psi \in \mathcal{L}_{\mathrm{FC}}$ that $\psi \in \mathcal{F C}(\phi \vee \psi)$. Since $\phi \notin K$, we have by $(E E 4)$ that $\phi<\psi$. Then we have by $\left(C_{\mathrm{FC}} \dot{-}\right)$ that $\psi \in K \dot{-} \phi$. Consequently, $\psi \in K$ and $\psi \in K \dot{-} \phi$ imply $K \subseteq K \dot{-} \phi$.

$(F \dot{-} 4)$ : By Definition 2, we have for each $\sigma \in \mathcal{F C}(\phi \vee \phi), \sigma \vdash_{\mathrm{FC}} \phi$. Thus we have by $(E E 2)$ that $\phi \nless \sigma$. Then it follows from $\nvdash_{\mathrm{FC}} \phi$ and $\left(C_{\mathrm{FC}} \dot{-}\right)$ that $\phi \notin K \dot{-} \phi$.

$(F \dot{-} d e)$ : Suppose $\psi \in K \backslash K \dot{-} \phi$. Then $\nvdash_{\mathrm{FC}} \phi$, for otherwise $\psi \in K \dot{-} \phi$ follows from $\left(C_{\mathrm{FC}} \dot{-}\right)$. It follows from $\nvdash_{\mathrm{FC}} \phi, \psi \in K \backslash K \dot{-} \phi$ and $\left(C_{\mathrm{FC}} \dot{-}\right)$ that $\phi \nless \sigma$ for all $\sigma \in \mathcal{F C}(\phi \vee \psi)$. For any such $\sigma$ we have $\phi \vee \sigma \vdash_{\mathrm{F}} \phi \vee \psi$ which means $\mathcal{F C}(\phi \vee \sigma) \subseteq \mathcal{F C}(\phi \vee \psi)$. Thus for any such $\sigma$, we have $\phi \nless \delta$ for all $\delta \in \mathcal{F C}(\phi \vee \sigma)$. Then it follows from $\left(C_{\mathrm{FC}} \dot{-}\right)$ that, for any such $\sigma, \sigma \notin K \dot{-} \phi$.

$(F-6)$ : Suppose $C n_{\mathrm{FC}}(\phi)=C n_{\mathrm{FC}}(\psi)$. Then $C n_{\mathrm{F}}(\phi \vee \delta)=C n_{\mathrm{F}}(\psi \vee \delta)$ for any $\delta \in \mathcal{L}_{\mathrm{FC}}$. Thus it follows from Definition 2 that $\mathcal{F C}(\phi \vee \delta)=\mathcal{F C}(\psi \vee \delta)$ for any $\delta \in \mathcal{L}_{\mathrm{FC}}$. Then $K \dot{-} \phi=K \dot{-} \psi$ is an immediate consequence of $\left(C_{\mathrm{FC}} \dot{-}\right)$.

$(F \dot{-r e})$ : Suppose $\psi \in K \dot{-} \phi$. Then we have by $\left(C_{\mathrm{FC}} \dot{-}\right)$ that either $\vdash_{\mathrm{FC}} \phi$ or there is $\sigma \in \mathcal{F C}(\phi \vee \psi)$ such that $\phi<\sigma$. For the former case, we have by Definition 2 that $\phi \in \mathcal{F C}(\phi \vee \psi)$. Then since $(F \dot{-} 1)$ gives $K \dot{-} \phi \wedge \phi=C n_{\mathrm{FC}}(K \dot{-} \phi \wedge \phi)$, we have $\phi \in K \dot{-} \phi \wedge \phi$. For the latter case, since $\phi<\sigma$ we have by $(C \leq)$ that $\sigma \in K \dot{-} \phi \wedge \sigma$.

$(F \dot{-} c t)$ : Suppose $\psi \in K \dot{-} \phi \wedge \psi$. Then we have by $(C \leq)$ that $\phi<\psi$. Since $\phi \wedge \delta \vdash_{\mathrm{FC}} \phi$, we have by $(E E 2)$ that $\phi \wedge \delta \leq \phi$. Then it follows from $(E E 1)$ and $\phi<\psi$ that $\phi \wedge \delta<\psi$ which implies by $(C \leq)$ that $\psi \in K \dot{-} \phi \wedge \psi \wedge \delta$. 
$(F \dot{-8})$ : Suppose $\phi \notin K \dot{-} \phi \wedge \psi$. Then $\nvdash_{\mathrm{FC}} \phi$, for otherwise $(F \dot{-} 1)$ implies $\phi \in K \dot{-} \phi \wedge \psi$. Since $\phi \notin K \dot{-} \phi \wedge \psi$, we have by $(C \leq)$ that $\phi \leq \psi$ which implies by Lemma 1 (part 6) that $\phi \leq \phi \wedge \psi$. Let $\delta \in K \dot{-} \phi \wedge \psi$. Then we have by $\left(C_{\mathrm{FC}} \dot{-}\right)$ that $\delta \in K$ and there is $\sigma \in \mathcal{F C}((\phi \wedge \psi) \vee \delta)$ such that $\phi \wedge \psi<\sigma$. Since $\sigma \in \mathcal{F C}((\phi \wedge \psi) \vee \delta)$, we have $\sigma \vdash_{\mathrm{F}}(\phi \wedge \psi) \vee \delta$. Since $(\phi \wedge \psi) \vee \delta \vdash_{\mathrm{F}} \phi \vee \delta$, we have $\sigma \vdash_{\mathrm{F}} \phi \vee \delta$. Then it follows from $\sigma \in \mathcal{L}_{\mathrm{FC}}$ that $\sigma \in \mathcal{F C}(\phi \vee \delta)$. Since $\phi \leq \phi \wedge \psi$ and $\phi \wedge \psi<\sigma$, we have by $(E E 1)$ that $\phi<\sigma$. It follows from $\sigma \in \mathcal{F C}(\phi \vee \delta)$, $\phi<\sigma$, and $\left(C_{\mathrm{FC}} \dot{-}\right)$ that $\delta \in K \dot{-} \phi$.

$\Leftarrow$ : Suppose $\dot{-}$ is a function that satisfies $(F \dot{-} 1)-(F \dot{-} 4),(F \dot{-d e}),(F \dot{-} 6),(F \dot{-} r e)$, $(F \dot{-} c t)$, and $(F \dot{-} 8)$. It suffices to show the $\mathrm{FC}$ epistemic entrenchment $\leq$ generated from $\dot{-}$ via $(C \leq)$ satisfies $(E E 1)-(E E 5)$ and $\left(C_{\mathrm{FC}} \dot{-}\right)$. By replacing the AGM contraction postulates with their corresponding $\left\langle\mathcal{L}_{\mathrm{FC}}, C n_{\mathrm{FC}}\right\rangle$ ones, the proof for satisfaction of $(E E 1)-(E E 5)$ is the same as that of Theorem 2.50 in (Hansson, 1999). We therefore only give the proof for $\left(C_{\mathrm{FC}} \dot{-}\right)$.

$\left(C_{\mathrm{FC}} \dot{-}\right): \Rightarrow$ : Suppose $\psi \in K \dot{-} \phi$. Then we have by $(F \dot{-} 2)$ that $\psi \in K$. Suppose $\forall_{\mathrm{FC}} \phi$, it suffices to show there is $\sigma \in \mathcal{F C}(\phi \vee \psi)$ such that $\phi<\sigma$. Since $\psi \in K \dot{-} \phi$, we have by $(F \dot{-} r e)$ and there is $\sigma \in \mathcal{F C}(\phi \vee \psi)$ such that $\sigma \in K \dot{-} \phi \wedge \sigma$. Then it follows from $(C \leq)$ and $\nvdash_{\mathrm{FC}} \phi \wedge \sigma$ that $\phi<\sigma$.

$\Leftarrow$ : Suppose $\psi \in K$. We need to show if $\vdash_{\mathrm{FC}} \phi$ or there is $\sigma \in \mathcal{F C}(\phi \vee \psi)$ such that $\phi<\sigma$, then $\psi \in K \dot{-} \phi$. Suppose $\vdash_{\mathrm{FC}} \phi$. Then $\vdash_{\mathrm{FC}} \phi \vee \psi$ which implies by Definition 2 that $\phi \in \mathcal{F C}(\phi \vee \psi)$. Then we have by $(F \dot{-} 1)$ that $\phi \in K \dot{-} \phi$. It then follows from $\psi \in K$ and the contrapositive of $(F \dot{-} d e)$ that $\psi \in K \dot{-} \phi$. Now suppose $\nvdash_{\mathrm{FC}} \phi$ and there is $\sigma \in \mathcal{F C}(\phi \vee \psi)$ such that $\phi<\sigma$. Then it follows from $(C \leq)$ and $\nvdash_{\mathrm{FC}} \phi \wedge \sigma$ that $\sigma \in K \dot{-} \phi \wedge \sigma$. Then we have by $(F \dot{-} 4)$ that $\phi \notin K \dot{-} \phi \wedge \sigma$ which implies by $(F \dot{-} 8)$ that $K \dot{-} \phi \wedge \sigma \subseteq K \dot{-} \phi$. Since $\sigma \in K \dot{-} \phi \wedge \sigma$, we have $\sigma \in K \dot{-} \phi$. Finally it follows from $\psi \in K$ and the contrapositive of $(F \dot{-}$ de $)$ that $\psi \in K \dot{-} \phi$.

\section{Proof of Theorem 3}

Suppose - is an FC entrenchment-based contraction function and the FC epistemic entrenchment associated with $K$ is $\leq$. Let $\delta \in(K \dot{-} \phi) \cap(K \dot{-} \psi)$. If $\vdash_{\mathrm{FC}} \phi$, then it follows from $(F \dot{-} 6)$ that $K \dot{-}(\phi \wedge \psi)=K \dot{-} \psi$. Thus $\delta \in K \dot{-}(\phi \wedge \psi)$. We can show the result in a similar way for when we have $\vdash_{\mathrm{FC}} \psi$. So suppose $\nvdash_{\mathrm{FC}} \phi$ and $\nvdash_{\mathrm{FC}} \psi$. It follows from $(E E 2)$ that $\phi \wedge \psi \leq \phi$ and $\phi \wedge \psi \leq \psi$. Since $\delta \in K \dot{-} \phi$, we have by $\left(C_{\mathrm{FC}} \dot{-}\right)$ that $\delta \in K$ and there is $\sigma_{1} \in \mathcal{F C}(\phi \vee \delta)$ such that $\phi<\sigma_{1}$. We then conclude from $(E E 1)$ and $\phi \wedge \psi \leq \phi$ that $\phi \wedge \psi<\sigma_{1}$. In a similar way we can show that there is $\sigma_{2} \in \mathcal{F C}(\psi \vee \delta)$ such that $\phi \wedge \psi<\sigma_{2}$. By Lemma 1 (part 5), we can deduce from $\phi \wedge \psi<\sigma_{1}$ and $\phi \wedge \psi<\sigma_{2}$ that $\phi \wedge \psi<\sigma_{1} \wedge \sigma_{2}$. Since $\sigma_{1} \in \mathcal{F C}(\phi \vee \delta)$ and $\sigma_{2} \in \mathcal{F C}(\psi \vee \delta)$, we have by Definition 2 that $\sigma_{1} \wedge \sigma_{2} \vdash_{\mathrm{F}}(\phi \vee \delta) \wedge(\psi \wedge \delta)$ which implies $\sigma_{1} \wedge \sigma_{2} \vdash_{\mathrm{F}}(\phi \wedge \psi) \vee \delta$. Then it follows from $\sigma_{1} \wedge \sigma_{2} \in \mathcal{L}_{\mathrm{FC}}$ that $\sigma_{1} \wedge \sigma_{2} \in \mathcal{F C}((\phi \wedge \psi) \vee \delta)$. Finally, it follows from $\delta \in K$, $\sigma_{1} \wedge \sigma_{2} \in \mathcal{F C}((\phi \wedge \psi) \vee \delta), \phi \wedge \psi<\sigma_{1} \wedge \sigma_{2}$ and $\left(C_{\mathrm{FC}} \dot{-}\right)$ that $\delta \in K \dot{-}(\phi \wedge \psi)$.

\section{Proof of Proposition 4}

$\Rightarrow$ : Suppose cut $_{\leq}(\neg \phi \vee \psi) \subset$ cut $_{\leq}(\neg \phi)$. Then there is $\gamma \in$ cut $_{\leq}(\neg \phi)$ such that $\{\sigma \in$ $K \mid \gamma \leq \sigma\} \vdash_{\mathrm{F}} \neg \phi \vee \psi$. Let $\bigwedge\{\sigma \in K \mid \gamma \leq \sigma\}$ be $\delta$. Then $\delta \vdash_{\mathrm{F}} \neg \phi \vee \psi$ which means $\delta \in \mathcal{F C}(\neg \phi \vee \psi)$. Also it follows from $(E E 3)$ that $\delta \leq \gamma$ and $\gamma \leq \delta$ which means $\delta \in$ cut $_{\leq}(\neg \phi)$. Since $\sigma \notin$ cut $_{\leq}(\neg \phi)$ for all $\sigma \in \mathcal{F C}(\neg \phi)$, we have $\sigma<\delta$ for all $\sigma \in \mathcal{F C}(\neg \phi)$. 
$\Leftarrow$ : Since $\neg \phi \vdash_{\mathrm{F}} \neg \phi \vee \psi$, it follows from the definition of cut that cut $_{\leq}(\neg \phi \vee \psi) \subseteq$ cut $_{\leq}(\neg \phi)$. It remains to show that there is $\delta \in c^{\prime} u t_{\leq}(\neg \phi)$ such that $\delta \notin c u t_{\leq}(\neg \phi \vee \psi)$. By the hypothesis, there is $\delta \in \mathcal{F C}(\neg \phi \vee \psi)$ such that $\sigma<\delta$ for all $\sigma \in \mathcal{F C}(\neg \phi)$. Since $\delta \vdash_{\mathrm{F}} \neg \phi \vee \psi$, $\delta \notin$ cut $_{\leq}(\neg \phi \vee \psi)$. Assume $\{\sigma \in K \mid \delta \leq \sigma\} \vdash_{\mathrm{F}} \neg \phi$ and let $\bigwedge\{\sigma \in K \mid \delta \leq \sigma\}$ be $\gamma$. Then $\gamma \vdash_{\mathrm{F}} \neg \phi$ which means $\gamma \in \mathcal{F C}(\neg \phi)$. But it follows from (EE3) that $\gamma \leq \delta$, a contradiction. Therefore $\{\sigma \in K \mid \delta \leq \sigma\} \nvdash_{\mathrm{F}} \neg \phi$ which means $\delta \in c u t_{\leq}(\neg \phi)$.

\section{Proof of Theorem 4}

Let $\mathcal{L}_{\mathrm{FC}}$ contains some tautologies and without loss of generality let one of them be $T$. Suppose $*$ is an $\mathrm{FC}$ revision function where the $\mathrm{FC}$ epistemic entrenchment associated with $K$ is $\leq$. We need to show $*$ satisfies $(F * 1)-(F * 8)$.

$(F * 1)$ : If $\vdash_{\mathrm{F}} \neg \phi$, then it follows from $\left(R_{\mathrm{FC}} *\right)$ that $K * \phi=K_{\perp}$. Since $K_{\perp}=C n_{\mathrm{FC}}\left(K_{\perp}\right)$, we have $K * \phi=C n_{\mathrm{FC}}(K * \phi)$. So suppose $\nvdash_{\mathrm{F}} \neg \phi$ and $K * \phi \vdash_{\mathrm{FC}} \psi$. By the compactness of FC logic, there is a finite subset $\left\{\theta_{1}, \ldots, \theta_{n}\right\}$ of $K * \phi$ such that $\left\{\theta_{1}, \ldots, \theta_{n}\right\} \vdash_{\mathrm{FC}} \psi$. Then it follows from $\left(R_{\mathrm{FC}} *\right)$ that there is $\delta_{i} \in \mathcal{F C}\left(\neg \phi \vee \theta_{i}\right)$ such that $\sigma<\delta_{i}$ for all $\sigma \in \mathcal{F C}(\neg \phi)$, where $1 \leqslant i \leqslant n$. Thus we have by Lemma 1 (part 5) that $\sigma<\delta_{1} \wedge \cdots \wedge \delta_{n}$ for all $\sigma \in \mathcal{F C}(\neg \phi)$. Since $\delta_{1} \wedge \cdots \wedge \delta_{n} \vdash_{\mathrm{F}}\left(\neg \phi \vee \theta_{1}\right) \wedge \cdots \wedge\left(\neg \phi \vee \theta_{n}\right)$ and $\left(\neg \phi \vee \theta_{1}\right) \wedge \cdots \wedge\left(\neg \phi \vee \theta_{n}\right) \vdash_{\mathrm{F}} \neg \phi \wedge \psi$, we have $\delta_{1} \wedge \cdots \wedge \delta_{n} \vdash_{\mathrm{F}} \neg \phi \wedge \psi$. Then it follows from $\delta_{1} \wedge \cdots \wedge \delta_{n} \in \mathcal{L}_{\mathrm{FC}}$ that $\delta_{1} \wedge \cdots \wedge \delta_{n} \in \mathcal{F C}(\neg \phi \vee \psi)$. Finally it follows from $\delta_{1} \wedge \cdots \wedge \delta_{n} \in \mathcal{F C}(\neg \phi \vee \psi), \sigma<\delta_{1} \wedge \cdots \wedge \delta_{n}$ for all $\sigma \in \mathcal{F C}(\neg \phi)$, and $\left(R_{\mathrm{FC}} *\right)$ that $\psi \in K * \phi$.

$(F * 2)$ : If $K+\phi$ is inconsistent, then it follows from $(F * 1)$ that $K+\phi=K_{\perp}$ which implies $K * \phi \subseteq K+\phi$. So suppose $K+\phi$ is consistent. Then $K \nvdash_{\mathrm{F}} \neg \phi$ which implies $K \nvdash_{\mathrm{FC}} \sigma$ for all $\sigma \in \mathcal{F C}(\neg \phi)$. Thus $\sigma \notin K$ for all $\sigma \in \mathcal{F C}(\neg \phi)$. Suppose $\psi \in K * \phi$. Then we have by $\left(R_{\mathrm{FC}} *\right)$ that there is $\delta \in \mathcal{F C}(\neg \phi \vee \psi)$ such that $\sigma<\delta$ for all $\sigma \in \mathcal{F C}(\neg \phi)$. Since for all $\sigma \in \mathcal{F C}(\neg \phi), \sigma \notin K$ and $\sigma<\delta$, we have by $(E E 4)$ that $\delta \in K$. It follows from $\delta \in K$, $\delta \in \mathcal{F C}(\neg \phi \vee \psi)$, and Lemma 2 that $K+\phi \vdash \psi$ which means $\psi \in K+\phi$.

$(F * 3)$ : Suppose $K+\phi$ is consistent. Then as for $(F * 2)$ we have $\sigma \notin K$ for all $\sigma \in \mathcal{F C}(\neg \phi)$. Suppose $\psi \in K+\phi$. Then we have by Lemma 2 that there is $\delta \in \mathcal{F C}(\neg \phi \vee \psi)$ such that $K \vdash_{\mathrm{FC}} \delta$ which means $\delta \in K$. Then it follow from $(E E 4)$ that $\sigma<\delta$ for all $\sigma \in \mathcal{F C}(\neg \phi)$. Finally, it follows from $\delta \in \mathcal{F C}(\neg \phi \vee \psi), \sigma<\delta$ for all $\sigma \in \mathcal{F C}(\neg \phi)$, and $\left(R_{\mathrm{FC}} *\right)$ that $\psi \in K * \phi$.

$(F * 4)$ : If $\phi$ is inconsistent, then $\vdash_{\mathrm{F}} \neg \phi$. Thus it follows from $\left(R_{\mathrm{FC}} *\right)$ that $K * \phi=K_{\perp}$ which implies $\phi \in K * \phi$. So suppose $\phi$ is consistent. Then $\nvdash_{\mathrm{F}} \neg \phi$ and $\top \in \mathcal{F C}(\neg \phi \vee \phi)$. So we have by $(E E 5)$ that $\sigma<\top$ for all $\sigma \in \mathcal{F C}(\neg \phi)$. Then it follows from $\left(R_{\mathrm{FC}} *\right)$ that $\phi \in K * \phi$.

$(F * 5): \Leftarrow$ : Suppose $\phi$ is inconsistent. Then it follows from $(F * 1)$ and $(F * 4)$ that $K * \phi=K_{\perp}$.

$\Rightarrow$ : Suppose $\phi$ is consistent. Let $\psi \in K * \phi$. Then there is $\delta \in \mathcal{F C}(\neg \phi \vee \psi)$ such that $\sigma<\delta$ for all $\sigma \in \mathcal{F C}(\neg \phi)$. Assume there is $\theta \in \mathcal{F C}(\neg \psi)$ such that $\theta \in K * \phi$. Then there is $\delta^{\prime} \in \mathcal{F C}(\neg \phi \vee \theta)$ such that $\sigma<\delta^{\prime}$ for all $\sigma \in \mathcal{F C}(\neg \phi)$. Then it follows from Lemma 1 (part $5)$ that $\sigma<\delta \wedge \delta^{\prime}$ for all $\sigma \in \mathcal{F C}(\neg \phi)$ Since $(\neg \phi \vee \theta) \wedge(\neg \phi \vee \psi) \vdash_{\mathrm{F}} \neg \phi$, we have $\delta \wedge \delta^{\prime} \vdash_{\mathrm{F}} \neg \phi$. Then it follows from $\delta \wedge \delta^{\prime} \in \mathcal{L}_{\mathrm{FC}}$ that $\delta \wedge \delta^{\prime} \in \mathcal{F C}(\neg \phi)$ which means $\delta \wedge \delta^{\prime}<\delta \wedge \delta^{\prime}$, a contradiction. Thus there is no $\theta \in \mathcal{F C}(\neg \psi)$ such that $\theta \in K * \phi$ which means $K * \phi$ is consistent.

$(F * 6)$ : Follows immediately from $\left(R_{\mathrm{FC}} *\right)$. 
$(F * 7)$ : We let $\phi$ and $\psi$ be consistent and non-tautology, for otherwise the proof is trivial. Let $\gamma \in K * \phi \wedge \psi$. Then there is $\delta \in \mathcal{F C}(\neg \phi \vee \neg \psi \vee \gamma)$ such that $\sigma<\delta$ for all $\sigma \in \mathcal{F C}(\neg \phi \vee \psi)$. Since $\neg \phi \vdash_{F} \neg \phi \vee \neg \psi$, we have $\mathcal{F C}(\neg \phi) \subseteq \mathcal{F C}(\neg \phi \vee \psi)$. Thus $\sigma^{\prime}<\delta$ for all $\sigma^{\prime} \in \mathcal{F C}(\neg \phi)$. Since $\delta \vdash_{\mathrm{F}} \neg \phi \vee \delta$, we have $\delta \in \mathcal{F C}(\neg \phi \vee \delta)$. It follows from $\delta \in \mathcal{F C}(\neg \phi \vee \delta)$, $\sigma^{\prime}<\delta$ for all $\sigma^{\prime} \in \mathcal{F C}(\neg \phi)$, and $\left(R_{\mathrm{FC}} *\right)$ that $\delta \in K * \phi$. Then since $\phi \in K * \phi$ follows from $(F * 4)$, we have $\{\delta, \phi, \psi\} \subseteq(K * \phi)+\psi$. Finally it follows from $\{\delta, \phi, \psi\} \vdash_{\mathrm{FC}} \gamma$ and $(F * 1)$ that $\gamma \in(K * \phi)+\psi$.

$(F * 8)$ : We let $\phi$ and $\psi$ be consistent and non-tautology, for otherwise the proof is trivial. Let $\gamma \in K * \phi \wedge \psi$. Suppose $(K * \phi)+\psi$ is consistent. Then $K * \phi \nvdash_{\mathrm{F}} \neg \psi$. Since $\phi \in K * \phi$ follows from $(F * 4)$, we have $K * \phi \nvdash_{\mathrm{F}} \neg \phi \vee \neg \psi$, for otherwise $K * \phi \vdash_{\mathrm{F}} \neg \psi$. Thus $\sigma \notin K * \phi$ for all $\sigma \in \mathcal{F C}(\neg \phi \vee \neg \psi)$. Then it follows from $\left(R_{\mathrm{FC}} *\right)$ that for all $\sigma \in \mathcal{F C}(\neg \phi \vee \neg \psi)$, $\delta \in \mathcal{F C}(\neg \phi \vee \sigma)$ implies there is $\sigma^{\prime} \in \mathcal{F C}(\neg \phi)$ such that $\delta \leq \sigma^{\prime}$. Since $\sigma \in \mathcal{F C}(\neg \phi \vee \sigma)$ follows from $\sigma \vdash_{\mathrm{F}} \neg \phi \vee \sigma$, we have for all $\sigma \in \mathcal{F C}(\neg \phi \vee \neg \psi)$ there is $\sigma^{\prime} \in \mathcal{F C}(\neg \phi)$ such that $\sigma \leq \sigma^{\prime}$.

Let $\gamma \in(K * \phi)+\psi$. Then it follows from Lemma 2 that there is $\delta \in \mathcal{F C}(\neg \psi \vee \gamma)$ such that $K * \phi \vdash_{\mathrm{FC}} \delta$. So we have by $(F * 1)$ that $\delta \in K * \phi$ which implies there is $\delta^{\prime} \in \mathcal{F C}(\neg \phi \vee \delta)$ such that $\sigma^{\prime}<\delta^{\prime}$ for all $\sigma^{\prime} \in \mathcal{F C}(\neg \phi)$. Since $\neg \phi \vee \delta \vdash_{\mathrm{F}} \neg \phi \vee \neg \psi \vee \gamma$, we have $\mathcal{F C}(\neg \phi \vee \delta) \subseteq \mathcal{F C}(\neg \phi \vee \neg \psi \vee \gamma)$. So there is $\delta^{\prime} \in \mathcal{F C}(\neg \phi \vee \neg \psi \vee \gamma)$ such that $\sigma^{\prime}<\delta^{\prime}$ for all $\sigma^{\prime} \in \mathcal{F C}(\neg \phi)$. Then since for all $\sigma \in \mathcal{F C}(\neg \phi \vee \neg \psi)$ there is $\sigma^{\prime} \in \mathcal{F C}(\neg \phi)$ such that $\sigma \leq \sigma^{\prime}$, we have by $(E E 1)$ that $\sigma<\delta^{\prime}$ for all $\sigma \in \mathcal{F C}(\neg \phi \vee \neg \psi)$. It follows from $\delta^{\prime} \in \mathcal{F C}(\neg \phi \vee \neg \psi \vee \gamma)$, $\sigma<\delta^{\prime}$ for all $\sigma \in \mathcal{F C}(\neg \phi \vee \neg \psi)$, and $\left(R_{\mathrm{FC}} *\right)$ that $\gamma \in K * \phi \wedge \psi$.

\section{Proof of Proposition 6}

Let the underlying logic be $\left\langle\mathcal{L}_{\mathrm{H}}, C n_{\mathrm{H}}\right\rangle$. Suppose the function $*$ is an FC revision function where the FC epistemic entrenchment associated with $K$ is $\leq$. According to Theorem 2 and 3 in (Delgrande \& Peppas, 2015), model-based Horn revision is fully characterised by $(F * 1)-(F * 8)$ and the schema (Acyc). It suffices to show $*$ satisfies $(F * 1)-(F * 8)$ and $(A c y c)$. Since $\top \in \mathcal{L}_{\mathrm{H}}$, we have by Theorem 5 that $*$ satisfies $(F * 1)-(F * 8)$. Hence it remains to show $*$ satisfies (Acyc).

Suppose for $0 \leq i<n,\left(K * \phi_{i+1}\right)+\phi_{i}$ is consistent, and $\left(K * \phi_{0}\right)+\phi_{n}$ is consistent. By Theorem 7 , we have $\left(K * \phi_{i+1}\right)+\phi_{i}=\left(\right.$ cut $\left._{\leq}\left(\neg \phi_{i+1}\right)+\phi_{i+1}\right)+\phi_{i}$, which means $(K *$ $\left.\phi_{i+1}\right)+\phi_{i}=$ cut $_{\leq}\left(\neg \phi_{i+1}\right)+\phi_{i+1} \wedge \phi_{i}$. Since $\left(K * \phi_{i+1}\right)+\phi_{i}$ is consistent, it follows from Lemma 2 that cut $_{\leq}\left(\neg \phi_{i+1}\right) \forall_{\mathrm{F}} \neg \phi_{i+1} \vee \neg \phi_{i}$ which means cut $\leq\left(\neg \phi_{i+1}\right) \forall_{\mathrm{F}} \neg \phi_{i}$. Let $\delta \in$ cut $_{\leq}\left(\neg \phi_{i+1}\right)$. Then it follows from the definition of cut and $(E E 1)$ that $\{\sigma \in K \mid \delta \leq$ $\sigma\} \subseteq$ cut $_{\leq}\left(\neg \phi_{i+1}\right)$ which implies $\{\sigma \in K \mid \delta \leq \sigma\} \nvdash_{\mathrm{F}} \neg \phi_{i}$. Hence $\delta \in c u t_{\leq}\left(\neg \phi_{i}\right)$, which means cut $\leq\left(\neg \phi_{i+1}\right) \subseteq$ cut $_{\leq}\left(\neg \phi_{i}\right)$. Similarly, from $\left(K * \phi_{0}\right)+\phi_{n}$ is consistent, we can deduce cut $_{\leq}\left(\neg \phi_{0}\right) \subseteq$ cut $_{\leq}\left(\neg \phi_{n}\right)$. It follows from cut $\leq\left(\neg \phi_{i+1}\right) \subseteq$ cut $_{\leq}\left(\neg \phi_{i}\right)$ for $0 \leq i<n$ and cut $_{\leq}\left(\neg \phi_{0}\right) \subseteq \operatorname{cut}_{\leq}\left(\neg \phi_{n}\right)$ that cut $_{\leq}\left(\neg \phi_{1}\right)=\cdots=\operatorname{cut}_{\leq}\left(\neg \phi_{n}\right)$.

By Theorem 7 , we have $\left(K * \phi_{n}\right)+\phi_{0}=\left(c u\left(\neg \phi_{n}\right)+\phi_{n}\right)+\phi_{0}$ which means $\left(K * \phi_{n}\right)+\phi_{0}=$ cut $_{\leq}\left(\neg \phi_{n}\right)+\phi_{0} \wedge \phi_{n}$. Since cut $_{\leq}\left(\neg \phi_{n}\right)=$ cut $_{\leq}\left(\neg \phi_{0}\right)$, we have cut $\leq\left(\neg \phi_{0}\right)+\phi_{0} \wedge \phi_{n}=$ cut $\leq\left(\neg \phi_{n}\right)+\phi_{0} \wedge \phi_{n}$. Then it follows from Theorem 7 that $\left(K * \phi_{0}\right)+\phi_{n}=\left(K * \phi_{n}\right)+\phi_{0}$ which implies $\left(K * \phi_{n}\right)+\phi_{0}$ is consistent.

\section{Proof of Theorem 5}


Let $\mathcal{L}_{\mathrm{FC}}$ contains some tautologies and for all $\phi \in \mathcal{L}_{\mathrm{FC}} \mathcal{F C}(\neg \phi)$ is finite up to logical equivalence. Suppose $*$ is a contraction generated revision function and the $\mathrm{FC}$ contraction that generates $*$ is - where the $\mathrm{FC}$ epistemic entrenchment associated with $K$ is $\leq$. We need to show $*$ satisfies $(F * 1)-(F * 8)$. The proofs for $(F * 1)-(F * 6)$ are trivial.

For $(F * 7)$, let $\mathcal{F C}(\neg \phi) \neq \emptyset$ for otherwise the proof is trivial. Then it follows from the definition of contraction generated revision that

$$
K *(\phi \wedge \psi)=\bigcap_{\sigma \in \mathcal{F} \mathcal{C}(\neg \phi \vee \neg \psi)}(K \dot{-} \sigma)+\phi \wedge \psi
$$

and

$$
(K * \phi)+\psi=\left(\bigcap_{\sigma \in \mathcal{F C}(\neg \phi)}(K \dot{-} \sigma)+\phi\right)+\psi=\bigcap_{\sigma \in \mathcal{F} \mathcal{C}(\neg \phi)}(K \dot{-} \sigma)+\phi \wedge \psi .
$$

Since $\neg \phi \vdash_{\mathrm{F}} \neg \phi \vee \neg \psi$, we have $\mathcal{F C}(\neg \phi) \subseteq \mathcal{F C}(\neg \phi \vee \neg \psi)$ which implies

$$
\bigcap_{\sigma \in \mathcal{F} \mathcal{C}(\neg \phi \vee \neg \psi)}(K \dot{-} \sigma) \subseteq \bigcap_{\sigma \in \mathcal{F} \mathcal{C}(\neg \phi)}(K \dot{-} \sigma)
$$

Hence,

$$
\bigcap_{\sigma \in \mathcal{F} \mathcal{C}(\neg \phi \vee \neg \psi)}(K \dot{-} \sigma)+\phi \wedge \psi \subseteq \bigcap_{\sigma \in \mathcal{F} \mathcal{C}(\neg \phi)}(K \dot{-} \sigma)+\phi \wedge \psi .
$$

For $(F * 8)$, we let $\phi$ and $\psi$ be consistent and non-tautological, and there is $\sigma \in \mathcal{F C}(\neg \phi)$ such that $\sigma \in K$, for otherwise the proof is trivial. Suppose $(K * \phi)+\psi$ is consistent. Then $(K * \phi) \nvdash_{\mathrm{F}} \neg \psi$. Since $K * \phi=\bigcap_{\sigma \in \mathcal{F C}(\neg \phi)}(K \dot{-} \sigma)+\phi$, it follows from $(K * \phi) \nvdash_{\mathrm{F}} \neg \psi$ and Lemma 2 that there is no $\pi \in \mathcal{F C}(\neg \phi \vee \neg \psi)$ such that $\pi \in \bigcap_{\sigma \in \mathcal{F C}(\neg \phi)}(K \dot{-} \sigma)$. Hence for every $\pi \in \mathcal{F C}(\neg \phi \vee \neg \psi)$ there is $\sigma \in \mathcal{F C}(\neg \phi)$ such that $\pi \notin K \dot{-} \sigma$. As $\pi \in \mathcal{F C}(\sigma \vee \pi)$, it then follows from $\left(C_{\mathrm{FC}} \dot{-}\right)$ that for every $\pi \in \mathcal{F C}(\neg \phi \vee \neg \psi)$ there is $\sigma \in \mathcal{F C}(\neg \phi)$ such that $\pi \leq \sigma$.

Suppose $\gamma \in(K * \phi)+\psi$. Then since $(K * \phi)+\psi=\bigcap_{\sigma \in \mathcal{F C}(\neg \phi)}(K \dot{-} \sigma)+\phi \wedge \psi$, we have by Lemma 2 that there is $\delta \in \mathcal{F C}(\neg \phi \vee \neg \psi \vee \gamma)$ such that $\delta \in \bigcap_{\sigma \in \mathcal{F C}(\neg \phi)}(K \dot{-} \sigma)$. Thus we have by $\left(C_{\mathrm{FC}} \dot{-}\right)$ that, for every $\sigma \in \mathcal{F C}(\neg \phi)$, there is $\delta^{\prime} \in \mathcal{F C}(\sigma \vee \delta)$ such that $\sigma<\delta^{\prime}$. Since $\sigma \vee \delta \vdash_{\mathrm{F}} \neg \phi \vee \neg \psi \vee \gamma$, we have $\mathcal{F C}(\sigma \vee \delta) \subseteq \mathcal{F C}(\neg \phi \vee \neg \psi \vee \gamma)$. Thus for every $\sigma \in \mathcal{F C}(\neg \phi)$, there is $\delta^{\prime} \in \mathcal{F C}(\neg \phi \vee \neg \psi \vee \gamma)$ such that $\sigma<\delta^{\prime}$. Since $\mathcal{F C}(\neg \phi)$ is finite up to logical equivalence, there is a $\sigma \in \mathcal{F C}(\neg \phi)$ such that $\sigma^{\prime} \leq \sigma$ for all $\sigma^{\prime} \in \mathcal{F C}(\neg \phi)$ and there is a $\delta^{\prime} \in \mathcal{F C}(\neg \phi \vee \neg \psi \vee \gamma)$ such that $\sigma<\delta^{\prime}$. Then for this $\delta^{\prime}$, we have by $(E E 1)$ and $(E E 4)$ that $\sigma<\delta^{\prime}$ for all $\sigma \in \mathcal{F C}(\neg \phi)$ and $\delta^{\prime} \in K$. Also, as for every $\pi \in \mathcal{F C}(\neg \phi \vee \neg \psi)$, there is $\sigma \in \mathcal{F C}(\neg \phi)$ such that $\pi \leq \sigma$, we have $\pi<\delta^{\prime}$ for all $\pi \in \mathcal{F C}(\neg \phi \vee \neg \psi)$. Since $\delta^{\prime} \in \mathcal{F C}\left(\pi \vee \delta^{\prime}\right)$ and $\delta^{\prime} \in K$, it then follows from $\left(C_{\mathrm{FC}} \dot{-}\right)$, that

$$
\delta^{\prime} \in \bigcap_{\pi \in \mathcal{F} \mathcal{C}(\neg \phi \vee \neg \psi)}(K \dot{-} \pi) .
$$

Since

$$
K * \phi \wedge \psi=\bigcap_{\pi \in \mathcal{F} \mathcal{C}(\neg \phi \vee \neg \psi)}(K \dot{-} \pi)+\phi \wedge \psi
$$


it then follows from Lemma 2 that $\gamma \in K * \phi \wedge \psi$.

\section{Proof of Theorem 6}

Let $\mathcal{L}_{\mathrm{FC}}$ contains some tautologies and for all $\phi \in \mathcal{L}_{\mathrm{FC}} \mathcal{F C}(\neg \phi)$ is finite up to logical equivalence. Let $*$ and $\dot{-}$ be an $\mathrm{FC}$ revision function and an $\mathrm{FC}$ contraction function respectively, and their determining $\mathrm{FC}$ epistemic entrenchments are identical for all $K$. Let $\mathcal{F C}(\neg \phi) \neq \emptyset$ for otherwise the proof is trivial. Then it suffices to show

$$
K * \phi=\bigcap_{\sigma \in \mathcal{F} \mathcal{C}(\neg \phi)}(K \dot{-} \sigma)+\phi
$$

We let $\phi$ be consistent and there is $\sigma \in \mathcal{F C}(\neg \phi)$ such that $\sigma \in K$, for otherwise the proof is trivial.

$\subseteq$ : Let $\psi \in K * \phi$. Then there is $\delta \in \mathcal{F C}(\neg \phi \vee \psi)$ such that $\sigma<\delta$ for all $\sigma \in \mathcal{F C}(\neg \phi)$. It then follows from $(E E 4)$ that $\delta \in K$. Since $\delta \vdash_{\mathrm{F}} \sigma \vee \delta$, we have $\delta \in \mathcal{F C}(\sigma \vee \delta)$ for every $\sigma \in \mathcal{F C}(\neg \phi)$. It then follows from $\left(C_{\mathrm{FC}} \dot{-}\right)$ that $\delta \in K \dot{-} \sigma$ for every $\sigma \in \mathcal{F C}(\neg \phi)$, which implies $\delta \in \bigcap_{\sigma \in \mathcal{F} \mathcal{C}(\neg \phi)}(K \dot{-} \sigma)$. Finally, we have by Lemma 2 that $\psi \in \bigcap_{\sigma \in \mathcal{F C}(\neg \phi)}(K \dot{-} \sigma)+\phi$.

?: Let $\psi \in \bigcap_{\sigma \in \mathcal{F C}(\neg \phi)}(K \dot{-} \sigma)+\phi$. Then we have by Lemma 2 that there is $\delta \in$ $\mathcal{F C}(\neg \phi \vee \psi)$ such that $\delta \in \bigcap_{\sigma \in \mathcal{F C}(\neg \phi)}(K \dot{-} \sigma)$. Thus for every $\sigma \in \mathcal{F C}(\neg \phi)$, it follows from $\left(C_{\mathrm{FC}} \dot{-}\right)$ that there is $\delta^{\prime} \in \mathcal{F C}(\delta \vee \sigma)$ such that $\sigma<\delta^{\prime}$. For every $\sigma \in \mathcal{F C}(\neg \phi)$, $\delta \vee \sigma \vdash_{\mathrm{F}} \neg \phi \vee \psi$ implies $\mathcal{F C}(\delta \vee \sigma) \subseteq \mathcal{F C}(\neg \phi \vee \psi)$, therefore we have, for every $\sigma \in \mathcal{F C}(\neg \phi)$, there is $\delta^{\prime} \in \mathcal{F C}(\neg \phi \vee \psi)$ such that $\sigma<\delta^{\prime}$. Since $\mathcal{F C}(\neg \phi)$ is finite up to logical equivalence, there is a $\sigma \in \mathcal{F C}(\neg \phi)$ such that $\sigma^{\prime} \leq \sigma$ for all $\sigma^{\prime} \in \mathcal{F C}(\neg \phi)$ and there is a $\delta^{\prime} \in \mathcal{F C}(\neg \phi \vee \psi)$ such that $\sigma<\delta^{\prime}$. Then for this $\delta^{\prime}$, we have by $(E E 1)$ and $(E E 4)$ that $\sigma<\delta^{\prime}$ for all $\sigma \in \mathcal{F C}(\neg \phi)$ and $\delta^{\prime} \in K$. Finally, it follows from $\left(R_{\mathrm{FC}} *\right)$ that $\psi \in K * \phi$.

\section{Proof of Theorem 7}

Let - and $\dot{-}_{w}$ be an $\mathrm{FC}$ contraction function and an $\mathrm{FC}$ withdrawal function respectively, and their determining FC epistemic entrenchments are identical for all $K$. It suffices to show

$$
\bigcap_{\sigma \in \mathcal{F} \mathcal{C}(\neg \phi)}(K \dot{-} \sigma)+\phi=\bigcap_{\sigma \in \mathcal{F} \mathcal{C}(\neg \phi)}\left(K \dot{ }_{w} \sigma\right)+\phi .
$$

: Suppose the FC epistemic entrenchment associated with $K$ is $\leq$ and $\psi \in K \dot{-}_{w} \sigma$ for all $\sigma \in \mathcal{F C}(\neg \phi)$. Then it follows from $\left(W_{\mathrm{FC}} \dot{-}\right)$ that $\psi \in K$ and $\sigma<\psi$ for all $\sigma \in \mathcal{F C}(\neg \phi)$. Since $\psi \in \mathcal{F C}(\sigma \vee \psi)$, it then follows from $\left(C_{\mathrm{FC}} \dot{-}\right)$ that $\psi \in K \dot{-} \sigma$ for all $\sigma \in \mathcal{F C}(\neg \phi)$. Therefore $\bigcap_{\sigma \in \mathcal{F C}(\neg \phi)}(K \dot{-} \sigma) \supseteq \bigcap_{\sigma \in \mathcal{F C}(\neg \phi)}\left(K \dot{-}_{w} \sigma\right)$, which means $\bigcap_{\sigma \in \mathcal{F C}(\neg \phi)}(K \dot{-} \sigma)+\phi \supseteq$ $\bigcap_{\sigma \in \mathcal{F C}(\neg \phi)}\left(K \dot{-}_{w} \sigma\right)+\phi$.

$\subseteq$ : Suppose $\psi \in \bigcap_{\sigma \in \mathcal{F C}(\neg \phi)}(K \dot{-} \sigma)+\phi$. Then it follows from Lemma 2 that there is $\delta \in \mathcal{F C}(\neg \phi \vee \psi)$ such that $\delta \in \bigcap_{\sigma \in \mathcal{F C}(\neg \phi)}(K \dot{-} \sigma)$. It then follows from $\left(C_{\mathrm{FC}} \dot{-}\right)$ that, for every $\sigma \in \mathcal{F C}(\neg \phi)$, there is $\delta^{\prime} \in \mathcal{F C}(\sigma \vee \delta)$ such that $\sigma<\delta^{\prime}$. Since $\sigma \vee \delta \vdash_{\mathrm{F}} \neg \phi \vee \psi$, we have, for every $\sigma \in \mathcal{F C}(\neg \phi), \mathcal{F C}(\sigma \vee \delta) \subseteq \mathcal{F C}(\neg \phi \vee \psi)$. Therefore, for every $\sigma \in \mathcal{F C}(\neg \phi)$ there is $\delta \in \mathcal{F C}(\neg \phi \vee \psi)$ such that $\sigma<\delta$. Since $\mathcal{F C}(\neg \phi)$ is finite, there is a $\sigma \in \mathcal{F C}(\neg \phi)$ such that $\sigma^{\prime} \leq \sigma$ for all $\sigma^{\prime} \in \mathcal{F C}(\neg \phi)$. For this $\sigma$ there is $\delta \in \mathcal{F C}(\neg \phi \vee \psi)$ such that $\sigma<\delta$. Hence for this $\delta$, we have $\sigma<\delta$ for all $\sigma \in \mathcal{F C}(\neg \phi)$. Also as $\delta \in K$ follows from (EE4), it then follows from $\left(W_{\mathrm{FC}} \dot{-}\right)$ that $\delta \in \bigcap_{\sigma \in \mathcal{F C}(\neg \phi)}(K \dot{-} \sigma)$ which means $\psi \in \bigcap_{\sigma \in \mathcal{F C}(\neg \phi)}\left(K \dot{-}_{w} \sigma\right)+\phi$. 


\section{Proof of Theorem 8}

Let $*$ and - be an $\mathrm{FC}$ revision function and an $\mathrm{FC}$ withdrawal function respectively, and their determining FC epistemic entrenchments are identical for all $K$. Let $\mathcal{F C}(\neg \phi) \neq \emptyset$ for otherwise the proof is trivial. Then it suffices to show

$$
K * \phi=\bigcap_{\sigma \in \mathcal{F} \mathcal{C}(\neg \phi)}(K \dot{-} \sigma)+\phi
$$

We let $\phi$ be consistent and there is $\sigma \in \mathcal{F C}(\neg \phi)$ such that $\sigma \in K$, for otherwise the proof is trivial.

$\subseteq$ : Let $\psi \in K * \phi$. Then there is $\delta \in \mathcal{F C}(\neg \phi \vee \psi)$ such that $\sigma<\delta$ for all $\sigma \in$ $\mathcal{F C}(\neg \phi)$. Since $\delta \in K$ follows from $(E E 4)$, we have by $\left(W_{\mathrm{FC}} \dot{-}\right)$ that $\delta \in K \dot{-} \sigma$ for every $\sigma \in \mathcal{F C}(\neg \phi)$, which implies $\delta \in \bigcap_{\sigma \in \mathcal{F C}(\neg \phi)}(K \dot{-} \sigma)$. Finally, we have by Lemma 2 that $\psi \in \bigcap_{\sigma \in \mathcal{F C}(\neg \phi)}(K \dot{-} \sigma)+\phi$.

?: Let $\psi \in \bigcap_{\sigma \in \mathcal{F C}(\neg \phi)}(K \dot{-} \sigma)+\phi$. Then we have by Lemma 2 that there is $\delta \in$ $\mathcal{F C}(\neg \phi \vee \psi)$ such that $\delta \in \bigcap_{\sigma \in \mathcal{F C}(\neg \phi)}(K \dot{-} \sigma)$. Then it follows from $\left(W_{\mathrm{FC}} \dot{-}\right)$ that $\sigma<\delta$ for all $\sigma \in \mathcal{F C}(\neg \phi)$. Finally, it follows from $\left(R_{\mathrm{FC}} *\right)$ that $\psi \in K * \phi$.

\section{Proof of Theorem 9}

Suppose $*$ is an FC revision function where the $\mathrm{FC}$ epistemic entrenchment associated with $K$ is $\leq$. Then it suffices show

$$
K * \phi=\text { cut }_{\leq}(\neg \phi)+\phi .
$$

$\subseteq$ : Let $\psi \in K * \phi$. Then there is $\delta \in \mathcal{F C}(\neg \phi \vee \psi)$ such that $\sigma<\delta$ for all $\sigma \in \mathcal{F C}(\neg \phi)$. Assume $\delta \notin$ cut $_{\leq}(\neg \phi)$. Then $\{\sigma \in K \mid \delta \leq \sigma\} \vdash_{\mathrm{F}} \neg \phi$. Let $\bigwedge\{\sigma \in K \mid \delta \leq \sigma\}$ be $\gamma$. Then $\gamma \in \mathcal{L}_{\mathrm{FC}}$ and $\gamma \vdash_{\mathrm{F}} \neg \phi$ which means there is $\sigma \in \mathcal{F C}(\neg \phi)$ such that $\gamma \vdash \sigma$. Then we have by $(E E 2)$ that $\gamma \leq \sigma$. Since it follows from $(E E 3)$ that $\delta \leq \gamma$, we have by $(E E 1)$ that $\delta \leq \sigma$, a contradiction. Therefore, $\delta \in$ cut $_{\leq}(\neg \phi)$ which implies by Lemma 2 that $\psi \in$ cut $_{\leq}(\neg \phi)+\phi$.

$\supseteq$ : Let $\psi \in$ cut $_{\leq}(\neg \phi)+\phi$. It then follows from Lemma 2 that there is $\delta \in \mathcal{F} \mathcal{C}(\neg \phi \vee \psi)$ such that $\delta \in c u t_{\leq}(\neg \phi)$. Then since $\sigma \notin c u t_{\leq}(\neg \phi)$ for all $\sigma \in \mathcal{F C}(\neg \phi)$, we have $\sigma<\delta$ for all $\sigma \in \mathcal{F C}(\neg \phi)$. Finally, it follows from $\left(R_{\mathrm{FC}} *\right)$ that $\psi \in K * \phi$.

\section{References}

Adaricheva, K., Sloan, R. H., \& Szörényi, B. (2012). Horn belief contraction: Remainders, envelopes and complexity. In Proceedings of the 13th International Conference on Principles of Knowledge Representation and Reasoning, pp. 107-115.

Alchourrón, C. E., Gärdenfors, P., \& Makinson, D. (1985). On the logic of theory change: Partial meet contraction and revision functions. The Journal of Symbolic Logic, 50(2), $510-530$.

Baader, F., Calvanese, D., McGuinness, D. L., Nardi, D., \& Patel-Schneider, P. F. (Eds.). (2003). The Description Logic Handbook: Theory, Implementation, and Applications. Cambridge University Press, New York, NY, USA. 
Booth, R., Meyer, T., Varzinczak, I. J., \& Wassermann, R. (2011). On the link between partial meet, kernel, and infra contraction and its application to Horn logic. Journal of Artificial Intelligence Research, 42, 31-53.

Calvanese, D., De Giacomo, G., Lembo, D., Lenzerini, M., \& Rosati, R. (2007). Tractable reasoning and efficient query answering in description logics: The DL-Lite family. Journal of Automatic Reasoning, 39(3), 385-429.

Creignou, N., Papini, O., Pichler, R., \& Woltran, S. (2014). Belief revision within fragments of propositional logic. Journal of Computer and System Sciences, 80(2), 427-449.

Delgrande, J. P., \& Jin, Y. (2012). Parallel belief revision: Revising by sets of formulas. Artificial Intelligence, 176(1), 2223-2245.

Delgrande, J. P., \& Peppas, P. (2015). Belief revision in Horn theories. Artificial Intelligence, 218, 1-22.

Delgrande, J. P., Peppas, P., \& Woltran, S. (2018). General belief revision. Journal of the Association for Computing Machinery, 65(5).

Delgrande, J. P., \& Wassermann, R. (2013). Horn clause contraction functions. Journal of Artificial Intelligence Research, 48, 475-551.

Fermé, E., Krevneris, M., \& Reis, M. (2008). An axiomatic characterization of ensconcement-based contraction. Journal of Logic and Computation, 18(5), 739-753.

Fuhrmann, A., \& Hansson, S. O. (1994). A survey of multiple contractions. Journal of Logic, Language and Information, 3(1), 39-74.

Gärdenfors, P. (1988). Knowledge in Flux: Modelling the Dynamics of Epistemic States. MIT Press.

Gärdenfors, P., \& Makinson, D. (1988). Revisions of knowledge systems using epistemic entrenchment. In Proceedings of the 2nd conference on Theoretical Aspects of Reasoning about Knowledge, pp. 83-95.

Grove, A. (1988). Two modellings for theory change. Journal of Philosophical Logic, 17(2), $157-170$.

Hansson, S. O. (1991). Belief contraction without recovery. Studia Logica, 50(2), 251-260.

Hansson, S. O. (1993). Changes of disjunctively closed bases. Journal of Logic, Language and Information, 2(4), 255-284.

Hansson, S. O. (1999). A Textbook of Belief Dynamics: Theory Change and Database Updating. Kluwer.

Harper, W. L. (1976). Rational conceptual change. PSA: Proceedings of the Biennial Meeting of the Philosophy of Science Association, 1976, 462-494.

Katsuno, H., \& Mendelzon, A. O. (1992). Propositional knowledge base revision and minimal change. Artificial Intelligence, 52(3), 263-294.

Kautz, H., \& Selman, B. (1996). Knowledge compilation and theory approximation. Journal of the ACM, 43, 193-224. 
Langlois, M., Sloan, R. H., Szörényi, B., \& Turán, G. (2008). Horn complements: Towards Horn-to-Horn belief revision. In Proceedings of the 23rd National Conference on Artificial Intelligence, pp. 466-471.

Levi, I. (1991). The Fixation of Beliefs and its Undoing. Cambridge University Press.

Lewis, D. (1973). Counterfactuals. Harvard University Press.

Makinson, D. (1987). On the status of the postulate of recovery in the logic of theory change. Journal of Philosophical Logic, 16(4), 383-394.

Peppas, P. (2008). Belief revision. In Handbook of Knowledge Representation, pp. 317-359. Elsevier Science.

Qi, G., \& Du, J. (2009). Model-based revision operators for terminologies in description logics. In Proceedings of the 21st International Joint Conferences on Artificial Intelligence (IJCAI-2009), pp. 891-897.

Qi, G., Haase, P., Huang, Z., Ji, Q., Pan, J. Z., \& Volker, J. (2008). A kernel revision operator for terminologies - algorithms and evaluation. In Proceedings of the 7th International Semantic Web Conference (ISWC-2008), pp. 419-434.

Ribeiro, M. M., Wassermann, R., Flouris, G., \& Antoniou, G. (2013). Minimal change: Relevance and recovery revisited. Artificial Intelligence, 201, 59-80.

Rott, H. (1991). Two methods of constructing contractions and revisions of knowledge systems. Journal of Philosophical Logic, 20(2), 149-173.

Rott, H. (1992). Preferential belief change using generalised epistemic entrenchment. Journal of Logic, Language and Information, 1(1), 45-78.

Rott, H., \& Pagnucco, M. (1999). Severe withdrawal (and recovery). Journal of Philosophical Logic, 28(5), 501-547.

Satoh, K. (1988). Nonmonotonic reasoning by minimal belief revision. In Proceedings of the International Conference on Fifth Generation Computer Systems, pp. 455-462.

Wang, Z., Wang, K., \& Topor, R. W. (2015a). DL-Lite ontology revision based on an alternative semantic characterization. ACM Transactions on Computational Logic, 16(4), 31:1-31:37.

Wang, Z., Wang, K., Zhuang, Z., \& Qi, G. (2015b). Instance-driven ontology evolution in dl-lite. In Proceedings of the 29th AAAI Conference on Artificial Intelligence, pp. $1656-1662$.

Williams, M.-A. (1994). On the logic of theory base change. In Proceedings of the European Workshop on Logics in Artificial Intelligence, pp. 86-105.

Wu, M., Zhang, D., \& Zhang, M. (2011). Language splitting and relevance-based belief change in horn logic. In Proceedings of the Twenty-Fifth AAAI Conference on Artificial Intelligence, pp. 268-273.

Zhuang, Z., \& Pagnucco, M. (2010). Horn contraction via epistemic entrenchment. In Proceedings of the 12th European Conference on Logics in Artificial Intelligence, pp. 339-351. 
Zhuang, Z., \& Pagnucco, M. (2011). Transitively Relational Partial Meet Horn Contraction. In Proceedings of the 22nd International Joint Conference on Artificial Intelligence, pp. 1132-1138.

Zhuang, Z., \& Pagnucco, M. (2012). Model Based Horn Contraction. In Proceedings of the 13th International Conference on Principles of Knowledge Representation and Reasoning, pp. 169-178.

Zhuang, Z., \& Pagnucco, M. (2014). Entrenchment-based Horn contraction. Journal of Artificial Intelligence Research, 51, 227-254.

Zhuang, Z., Pagnucco, M., \& Zhang, Y. (2013). Definability of Horn revision from Horn contraction. In Proceedings of the 23rd International Joint Conference on Artificial Intelligence, pp. 1205-1211.

Zhuang, Z., Pagnucco, M., \& Zhang, Y. (2017). Inter-definability of Horn contraction and Horn revision. Journal of Philosophical Logic, 46, 299-332.

Zhuang, Z., Wang, Z., Wang, K., \& Qi, G. (2014). Contraction and revision over DL-Lite TBoxes. In Proceedings of the 28th AAAI Conference on Atificial Intelligence, pp. 1149-1156.

Zhuang, Z., Wang, Z., Wang, K., \& Qi, G. (2016). DL-Lite contraction and revision. Journal of Artificial Intelligence Research, 56, 329-378. 